\title{
Frames2: A Package for Estimation in Dual Frame Surveys
}

\author{
by Antonio Arcos, David Molina, Maria Giovanna Ranalli and María del Mar Rueda
}

\begin{abstract}
Data from complex survey designs require special consideration with regard to estimation of finite population parameters and corresponding variance estimation procedures, as a consequence of significant departures from the simple random sampling assumption. In the past decade a number of statistical software packages have been developed to facilitate the analysis of complex survey data. All these statistical software packages are able to treat samples selected from one sampling frame containing all population units. Dual frame surveys are very useful when it is not possible to guarantee a complete coverage of the target population and may result in considerable cost savings over a single frame design with comparable precision. There are several estimators available in the statistical literature but no existing software covers dual frame estimation procedures. This gap is now filled by package Frames2. In this paper we highlight the main features of the package. The package includes the main estimators in dual frame surveys and also provides interval confidence estimation.
\end{abstract}

\section{Introduction}

Classic sampling theory usually assumes the existence of one sampling frame containing all finite population units. Then, a probability sample is drawn according to a given sampling design and information collected is used for estimation and inference purposes. In traditional 'design-based' inference the population data are regarded as fixed and the randomness comes entirely from the sampling procedure. The most used design-based estimator is the Horvitz-Thompson estimator that is unbiased for the population total if the sampling frame includes all population units, if all sampled units respond and if there is no measurement error. In the presence of auxiliary information, there exist several procedures to obtain more efficient estimators for population means and totals of variables of interest; in particular, customary ratio, regression, raking, post-stratified and calibration estimators. Several software packages have been developed to facilitate the analysis of complex survey data and implement some of these estimators as SAS (SAS Institute Inc., 2013), SPSS (IBM Corporation, 2013), Systat (Systat Software Inc., 2009), Stata (Stata Corporation, 2015), SUDAAN (Research Triangle Institute, 2013) and PCCarp (Fuller et al., 1989). CRAN hosts several R packages that include these design-based methods typically used in survey methodology to treat samples selected from one sampling frame (e.g. survey, Lumley 2014; sampling, Tillé and Matei 2012; laeken, Alfons et al. 2014 or TeachingSampling, Gutierrez Rojas 2014, among others). Templ (2014) provides a detailed list of packages that includes methods to analyse complex surveys.

In practice, the assumption that the sampling frame contains all population units is rarely met. Often, one finds that sampling from a frame which is known to cover approximately all units in the population is quite expensive while other frames (e.g. special lists of units) are available for cheaper sampling methods. However, the latter usually only cover an unknown or only approximately known fraction of the population. A common example of frame undercoverage is provided by telephone surveys. Estimation could be affected by serious bias due to the lack of a telephone in some households and the generalised use of mobile phones, which are sometimes replacing fixed (land) lines entirely. The potential for coverage error as a result of the exponential growth of the cell-phone only population has led to the development of dual-frame surveys. In these designs, a traditional sample from the landline frame is supplemented with an independent sample from a register of cell-phone numbers.

The dual frame sampling approach assumes that two frames are available for sampling and that, overall, they cover the entire target population. The most common situation is the one represented in Figure 1 where the two frames, say frame $A$ and frame $B$, show a certain degree of overlapping, so it is possible to distinguish three disjoint non-empty domains: domain $a$, containing units belonging to frame $A$ but not to frame $B$; domain $b$, containing units belonging to frame $B$ but not to frame $A$ and domain $a b$, containing units belonging to both frames. As an example, consider a telephone survey where both landline and cell phone lists are available; let $A$ be the landline frame and $B$ the cell phone frame. Then, it is possible to distinguish three types of individuals: landline only units, cell-only units and units with both landline and cell phone, which will compose domain $a, b$ and $a b$, respectively.

Nevertheless, one can face some other situations depending on the relative positions of the frames. For example, Figure 2 shows the case in which frame $B$ is totally included in frame $A$, that is, frame $B$ is a subset of frame $A$. Here domain $b$ is empty. We also may find scenarios where the two sampling frames exactly match, as depicted in Figure 3, where $a b$ is the only non-empty domain. Finally, the scenario where domain $a b$ is empty has no interest from a dual frame perspective, since it can be 


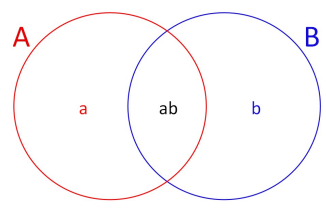

Figure 1: Two frames with overlapping.

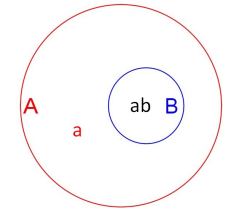

Figure 2: Frame $B$ is included in frame $A$.

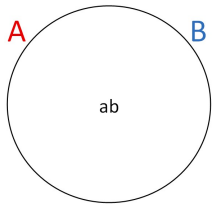

Figure 3: Frame $A$ and frame $B$ exactly match.

considered as a special case of stratified sampling.

Whatever the scenario, an appropriate choice of the frames results in a better coverage of the target population, which, in turn, leads to a better efficiency of estimators calculated from data from dual frame surveys. This point is particularly important when estimating parameters in rare or elusive populations, where undercoverage errors are usually due to the difficulty of finding individuals showing the characteristic under study when sampling from only one general frame. This issue can be dealt with by incorporating a second frame with a high density of members of the rare population so that the two frames are, together, now complete. Dual frame sampling as a method of improvement of efficiency may seem expensive and unviable, but it is not. In fact, Hartley (1962) notes that dual frame surveys can result in important cost savings in comparison with single frame ones with a comparable efficiency. As an additional interesting characteristic, dual frame methodology offers the researcher the possibility to consider different data collection procedures and/or different sampling designs, one for each frame. Dual frame surveys have gained much attention and became largely used by statistical agencies and private organizations to take advantage of these benefits.

Standard software packages for complex surveys cannot be used directly when the sample is obtained from a dual frame survey because the classical design-based estimators are severely biased and there is a underestimation of standard errors. Weighted analyses with standard statistical software, with certain modified weights, can yield correct point estimates of population parameters but still yield incorrect results for estimated standard errors. A number of authors have developed methods for estimating population means and totals from dual (or, more generally, multiple) frame surveys but most of these methods require ad-hoc software for their implementation. To the best of our knowledge, there is no software incorporating these estimation procedures for handling dual frame surveys.

Frames2 (Arcos et al., 2015) tries to fill this gap by providing functions for point and interval estimation from dual frame surveys. The paper is organized as follows. In the next section, we provide an overview of the main point estimators proposed so far in the dual frame context and review also jackknife variance estimation as a tool to compare efficiency for all of them. Subsequently, we present package Frames2, discussing guidelines that have been followed to construct it and presenting its principal functions and functionalities. We also provide examples to illustrate how the package works.

\section{Estimation in dual frame surveys}

Consider again the situation depicted in Figure 1. Assume we have a finite set of $N$ population units identified by integers, $\mathcal{U}=\{1, \ldots, k, \ldots, N\}$, and let $A$ and $B$ be two sampling frames, both can be incomplete, but it is assumed that together they cover the entire finite population.

Let $\mathcal{A}$ be the set of population units in frame $A$ and $\mathcal{B}$ the set of population units in frame $B$. The population of interest, $\mathcal{U}$, may be divided into three mutually exclusive domains, $a=\mathcal{A} \cap \mathcal{B}^{c}, b=$ $\mathcal{A}^{c} \cap \mathcal{B}$ and $a b=\mathcal{A} \cap \mathcal{B}$. Let $N, N_{A}, N_{B}, N_{a}, N_{b}$ and $N_{a b}$ be the number of population units in $\mathcal{U}, \mathcal{A}, \mathcal{B}, a, b, a b$, respectively.

Let $y$ be a variable of interest in the population and let $y_{k}$ be its value on unit $k$, for $k=1, \ldots, N$. The objective is to estimate the finite population total $Y=\sum_{k} y_{k}$ that can be written as

$$
Y=Y_{a}+Y_{a b}+Y_{b}
$$

where $Y_{a}=\sum_{k \in a} y_{k}, Y_{a b}=\sum_{k \in a b} y_{k}$ and $Y_{b}=\sum_{k \in b} y_{k}$. To this end, independent samples $s_{A}$ and $s_{B}$ are drawn from frame $A$ and frame $B$ of sizes $n_{A}$ and $n_{B}$, respectively. Unit $k$ in $\mathcal{A}$ has first-order inclusion probability $\pi_{k}^{A}=\operatorname{Pr}\left(k \in s_{A}\right)$ and unit $k$ in $\mathcal{B}$ has first-order inclusion probability $\pi_{k}^{B}=\operatorname{Pr}\left(k \in s_{B}\right)$.

From data collected in $s_{A}$, it is possible to compute one unbiased estimator of the total for each domain in frame $A, \hat{Y}_{a}$ and $\hat{Y}_{a b}^{A}$, as described below:

$$
\hat{Y}_{a}=\sum_{k \in s_{A}} \delta_{k}(a) d_{k}^{A} y_{k}, \quad \hat{Y}_{a b}^{A}=\sum_{k \in s_{A}} \delta_{k}(a b) d_{k}^{A} y_{k}
$$


where $\delta_{k}(a)=1$ if $k \in a$ and 0 otherwise, $\delta_{k}(a b)=1$ if $k \in a b$ and 0 otherwise and $d_{k}^{A}$ are the weights under the sampling design used in frame $A$, defined as the inverse of the first order inclusion probabilities, $d_{k}^{A}=1 / \pi_{k}^{A}$. Similarly, using information included in $s_{B}$, one can obtain an unbiased estimator of the total for domain $b$ and another one for domain $a b, \hat{Y}_{b}$ and $\hat{Y}_{a b}^{B}$, which can be expressed as

$$
\hat{Y}_{b}=\sum_{k \in s_{B}} \delta_{k}(b) d_{k}^{B} y_{k}, \quad \hat{Y}_{a b}^{B}=\sum_{k \in s_{B}} \delta_{k}(a b) d_{k}^{B} y_{k}
$$

with $\delta_{k}(b)=1$ if $k \in b$ and 0 otherwise, and $d_{k}^{B}$ the weights under the sampling design used in frame $B$ defined as the inverse of the first order inclusion probabilities, $d_{k}^{B}=1 / \pi_{k}^{B}$.

Different approaches for estimating the population total from dual frame surveys have been proposed in the literature. Hartley (1962) suggests the use of a parameter, $\theta$, to weight $\hat{Y}_{a b}^{A}$ and $\hat{Y}_{a b}^{B}$, providing the estimator

$$
\hat{Y}_{H}=\hat{Y}_{a}+\theta \hat{Y}_{a b}^{A}+(1-\theta) \hat{Y}_{a b}^{B}+\hat{Y}_{b}
$$

where $\theta \in[0,1]$. Hartley (1974) himself proved that

$$
\theta_{o p t}=\frac{V\left(\hat{Y}_{a b}^{B}\right)+\operatorname{Cov}\left(\hat{Y}_{b}, \hat{Y}_{a b}^{B}\right)-\operatorname{Cov}\left(\hat{Y}_{a}, \hat{Y}_{a b}^{A}\right)}{V\left(\hat{Y}_{a b}^{A}\right)+V\left(\hat{Y}_{a b}^{B}\right)}
$$

is the optimum value for $\theta$ so that variance of the estimator with respect to the design is minimized. In practice, $\theta_{\text {opt }}$ cannot be computed, since population variances and covariances involved in its calculation are unknown, so they must be estimated from sampling data. An estimator for the variance of $\hat{Y}_{H}$ can be computed, taking into account that samples from frame $A$ and frame $B$ are drawn independently, as follows

$$
\hat{V}\left(\hat{Y}_{H}\right)=\hat{V}\left(\hat{Y}_{a}\right)+\theta^{2} \hat{V}\left(\hat{Y}_{a b}^{A}\right)+\theta \widehat{\operatorname{Cov}}\left(\hat{Y}_{a}, \hat{Y}_{a b}^{A}\right)+(1-\theta)^{2} \hat{V}\left(\hat{Y}_{a b}^{B}\right)+\hat{V}\left(\hat{Y}_{b}\right)+(1-\theta) \widehat{\operatorname{Cov}}\left(\hat{Y}_{b}, \hat{Y}_{a b}^{B}\right),
$$

where hats denote suitable variance and covariance estimators.

Fuller and Burmeister (1972) introduce information from the estimation of overlap domain size, obtaining the following estimator

$$
\hat{Y}_{F B}=\hat{Y}_{a}+\hat{Y}_{b}+\beta_{1} \hat{Y}_{a b}^{A}+\left(1-\beta_{1}\right) \hat{Y}_{a b}^{B}+\beta_{2}\left(\hat{N}_{a b}^{A}-\hat{N}_{a b}^{B}\right),
$$

where $\hat{N}_{a b}^{A}=\sum_{k \in s_{A}} \delta_{k}(a b) d_{k}^{A}$ and $\hat{N}_{a b}^{B}=\sum_{k \in s_{B}} \delta_{k}(a b) d_{k}^{B}$. Fuller and Burmeister (1972) also show that

$$
\begin{aligned}
& {\left[\begin{array}{l}
\tilde{\beta}_{1} \\
\tilde{\beta}_{2}
\end{array}\right]=-\left[\begin{array}{cc}
V\left(\hat{Y}_{a b}^{A}-\hat{Y}_{a b}^{B}\right) & \operatorname{Cov}\left(\hat{Y}_{a b}^{A}-\hat{Y}_{a b}^{B}, \hat{N}_{a b}^{A}-\hat{N}_{a b}^{B}\right) \\
\operatorname{Cov}\left(\hat{Y}_{a b}^{A}-\hat{Y}_{a b}^{B}, \hat{N}_{a b}^{A}-\hat{N}_{a b}^{B}\right) & V\left(\hat{N}_{a b}^{A}-\hat{N}_{a b}^{B}\right)
\end{array}\right]^{-1}} \\
& \times\left[\begin{array}{c}
\operatorname{Cov}\left(\hat{Y}_{a}+\hat{Y}_{b}+\hat{Y}_{a b}^{B}, \hat{Y}_{a b}^{A}-\hat{Y}_{a b}^{B}\right) \\
\operatorname{Cov}\left(\hat{Y}_{a}+\hat{Y}_{b}+\hat{Y}_{a b}^{B}, \hat{N}_{a b}^{A}-\hat{N}_{a b}^{B}\right)
\end{array}\right]
\end{aligned}
$$

are the optimal values for $\beta_{1}$ and $\beta_{2}$ in the sense that they minimize the variance of the estimator. Again, $\tilde{\beta}_{1}$ and $\tilde{\beta}_{2}$ need to be estimated, since population values are not known in practice. An estimator for the variance of $\hat{Y}_{F B}$ is given by

$$
\hat{V}\left(\hat{Y}_{F B}\right)=\hat{V}\left(\hat{Y}_{a}\right)+\hat{V}\left(\hat{Y}_{B}\right)+\hat{\beta}_{1}\left(\widehat{\operatorname{Cov}}\left(\hat{Y}_{a}, \hat{Y}_{a b}^{A}\right)-\widehat{\operatorname{Cov}}\left(\hat{Y}_{B}, \hat{Y}_{a b}^{B}\right)\right)+\hat{\beta}_{2}\left(\widehat{\operatorname{Cov}}\left(\hat{Y}_{a}, \hat{N}_{a b}^{A}\right)-\widehat{\operatorname{Cov}}\left(\hat{Y}_{B}, \hat{N}_{a b}^{B}\right)\right),
$$

with $\hat{Y}_{B}=\hat{Y}_{b}+\hat{Y}_{a b}^{B}$.

Bankier (1986) and Kalton and Anderson (1986) combine all sampling units coming from the two frames, $s_{A}$ and $s_{B}$, trying to build a single sample as if it was drawn from only one frame. Sampling weights for the units in the overlap domain need, then, to be modified to avoid bias. These adjusted weights are

$$
\tilde{d}_{k}^{A}=\left\{\begin{array}{ll}
d_{k}^{A} & \text { if } k \in a \\
\left(1 / d_{k}^{A}+1 / d_{k}^{B}\right)^{-1} & \text { if } k \in a b
\end{array} \quad \text { and } \quad \tilde{d}_{k}^{B}= \begin{cases}d_{k}^{B} & \text { if } k \in b \\
\left(1 / d_{k}^{A}+1 / d_{k}^{B}\right)^{-1} & \text { if } k \in a b\end{cases}\right.
$$

or, summarizing,

$$
\tilde{d}_{k}=\left\{\begin{array}{ll}
d_{k}^{A} & \text { if } k \in a \\
\left(1 / d_{k}^{A}+1 / d_{k}^{B}\right)^{-1} & \text { if } k \in a b \\
d_{k}^{B} & \text { if } k \in b
\end{array} .\right.
$$

Hence, the estimator can be expressed in the form

$$
\hat{Y}_{B K A}=\sum_{k \in s_{A}} \tilde{d}_{k}^{A} y_{k}+\sum_{k \in s_{B}} \tilde{d}_{k}^{B} y_{k}=\sum_{k \in S} \tilde{d}_{k} y_{k}
$$


with $s=s_{A} \cup s_{B}$. Note that to compute this estimator, one needs to know, for units in the sample coming from the overlap domain, the inclusion probability under both sampling designs. Rao and Skinner (1996) propose the following unbiased estimator for the variance of the estimator

$$
\hat{V}\left(\hat{Y}_{B K A}\right)=\hat{V}\left(\sum_{k \in s_{A}} \tilde{z}_{k}^{A}\right)+\hat{V}\left(\sum_{k \in s_{B}} \tilde{z}_{k}^{B}\right),
$$

where $\tilde{z}_{k}^{A}=\delta_{k}(a) y_{k}+\left(1-\delta_{k}(a)\right) y_{k} \frac{\pi_{k}^{A}}{\pi_{k}^{A}+\pi_{k}^{B}}$ and $\tilde{z}_{k}^{B}=\delta_{k}(b) y_{k}+\left(1-\delta_{k}(b)\right) y_{k} \frac{\pi_{k}^{B}}{\pi_{k}^{A}+\pi_{k}^{B}}$.

When frame sizes, $N_{A}$ and $N_{B}$, are known, estimator (6) can be adjusted to increase efficiency through different procedures such as, for example, raking ratio (Bankier, 1986; Skinner, 1991). Applying the latter, one obtains a new estimator, usually called raking ratio (Skinner, 1991), which has the form

$$
\hat{Y}_{S F R R}=\frac{N_{A}-\hat{N}_{a b}^{r a k e}}{\hat{N}_{a}} \hat{Y}_{a}^{A}+\frac{N_{B}-\hat{N}_{a b}^{r a k e}}{\hat{N}_{b}} \hat{Y}_{b}^{B}+\frac{\hat{N}_{a b}^{r a k e}}{\hat{N}_{a b S}} \hat{Y}_{a b S},
$$

where $\hat{Y}_{a b S}=\sum_{k \in s_{A}} \tilde{d}_{k}^{A} \delta_{k}(a b) y_{k}+\sum_{k \in s_{B}} \tilde{d}_{k}^{B} \delta_{k}(a b) y_{k}, \hat{N}_{a b s}=\sum_{k \in s_{A}} \tilde{d}_{k}^{A} \delta_{k}(a b)+\sum_{k \in s_{B}} \tilde{d}_{k}^{B} \delta_{k}(a b), \hat{N}_{a}=$ $\sum_{k \in s_{A}} \delta_{k}(a), \hat{N}_{b}=\sum_{k \in s_{B}} \delta_{k}(b)$ and $\hat{N}_{a b}^{r a k e}$ is the smaller root of the quadratic equation $\hat{N}_{a b s} x^{2}-$ $\left(\hat{N}_{a b S}\left(N_{A}+N_{B}\right)+\hat{N}_{a S}^{A} \hat{N}_{b S}^{B}\right) x+\hat{N}_{a b S} N_{A} N_{B}=0$.

Skinner and Rao (1996) use a pseudo maximum likelihood approach to extend to complex designs the maximum likelihood estimator proposed by Fuller and Burmeister (1972) only for simple random sampling without replacement. The resulting estimator is given by

$$
\hat{Y}_{P M L}=\frac{N_{A}-\hat{N}_{a b}^{P M L}(\gamma)}{\hat{N}_{a}^{A}} \hat{Y}_{a}^{A}+\frac{N_{B}-\hat{N}_{a b}^{P M L}(\gamma)}{\hat{N}_{b}^{B}} \hat{Y}_{b}^{B}+\frac{\hat{N}_{a b}^{P M L}(\gamma)}{\gamma \hat{N}_{a b}^{A}+(1-\gamma) \hat{N}_{a b}^{B}}\left[\gamma \hat{Y}_{a b}^{A}+(1-\gamma) \hat{Y}_{a b}^{B}\right],
$$

where $\hat{N}_{a b}^{P M L}(\gamma)$ is the smallest of the roots of the quadratic equation $\left[\gamma / N_{B}+(1-\gamma) / N_{A}\right] x^{2}-[1+$ $\left.\gamma \hat{N}_{a b}^{A} / N_{B}+(1-\gamma) \hat{N}_{a b}^{B} / N_{A}\right] x+\gamma \hat{N}_{a b}^{A}+(1-\gamma) \hat{N}_{a b}^{B}=0$ and $\gamma \in(0,1)$. It is also shown that the following value for $\gamma$

$$
\gamma_{o p t}=\frac{\hat{N}_{a} N_{B} V\left(\hat{N}_{a b}^{B}\right)}{\hat{N}_{a} N_{B} V\left(\hat{N}_{a b}^{B}\right)+\hat{N}_{b} N_{A} V\left(\hat{N}_{a b}^{A}\right)}
$$

minimizes the variance of $\hat{Y}_{P M L}$. One can use the delta method to obtain a consistent estimator of the variance of this estimator of the form

$$
\hat{V}\left(\hat{Y}_{P M L}\right)=\hat{V}\left(\sum_{k \in s_{A}} \tilde{z}_{k}^{A}\right)+\hat{V}\left(\sum_{k \in s_{B}} \tilde{z}_{k}^{B}\right),
$$

where, in this case, $\tilde{z}_{k}^{A}=y_{k}-\frac{\hat{Y}_{a}}{\hat{N}_{a}}$ if $k \in a$ and $\tilde{z}_{k}^{A}=\hat{\gamma}_{o p t}\left(y_{k}-\frac{\hat{Y}_{a b}^{A}}{\hat{N}_{a b}^{A}}\right)+\hat{\lambda} \hat{\phi}$ if $k \in a b$, with $\hat{\gamma}_{o p t}$ an estimator of $\gamma_{o p t}$ in (10) obtained by replacing population quantities with their estimators, $\hat{\lambda}=$ $\frac{n_{A} / N_{A} \hat{Y}_{a}^{A}+n_{B} / N_{B} \hat{a}_{a b}^{B}}{n_{A} / N_{A} \hat{N}_{a b}^{A}+n_{B} / N_{B} \hat{N}_{a b}^{B}}-\frac{\hat{Y}_{a}}{\hat{N}_{a}}-\frac{\hat{Y}_{b}}{\hat{N}_{b}}$ and $\hat{\phi}=\frac{n_{A} \hat{N}_{b}}{n_{A} \hat{N}_{b}+n_{B} \hat{N}_{a}}$. Similary, one can define $\tilde{z}_{k}^{B}=y_{k}-\frac{\hat{Y}_{b}}{\hat{N}_{b}}$ if $k \in b$ and $\tilde{z}_{k}^{B}=\left(1-\hat{\gamma}_{o p t}\right)\left(y_{k}-\frac{\hat{\gamma}_{a b}^{B}}{\hat{N}_{a b}^{B}}\right)+\hat{\lambda}(1-\hat{\phi})$ if $k \in a b$.

More recently, Rao and Wu (2010) proposed a pseudo empirical likelihood estimator for the population mean based on poststratified samples. The estimator is computed as

$$
\hat{\bar{Y}}_{P E L}=\frac{N_{a}}{N} \hat{\bar{Y}}_{a}+\frac{\eta N_{a b}}{N} \hat{\bar{Y}}_{a b}^{A}+\frac{(1-\eta) N_{a b}}{N} \hat{\bar{Y}}_{a b}^{B}+\frac{N_{b}}{N} \hat{\bar{Y}}_{b}
$$

where, in this case, $\hat{\bar{Y}}_{a}=\sum_{k \in s_{A}} \hat{p}_{a k} y_{k} \delta_{k}(a), \hat{\bar{Y}}_{a b}^{A}=\sum_{k \in s_{A}} \hat{p}_{a b k}^{A} y_{k} \delta_{k}(a b), \hat{\bar{Y}}_{a b}^{B}=\sum_{k \in s_{B}} \hat{p}_{a b k}^{B} y_{k} \delta_{k}(a b)$ and $\hat{\bar{Y}}_{b}=\sum_{k \in s_{B}} \hat{p}_{b k} y_{k} \delta_{k}(b)$ with $\hat{p}_{a k}, \hat{p}_{a b k}^{A}, \hat{p}_{a b k}^{B}$ and $\hat{p}_{b k}$ the weights resulting from maximizing the pseudo empirical likelihood procedure under a set of constraints (see Rao and Wu 2010 for details). Furthermore, $\eta \in(0,1)$. In this case, it is assumed that $N_{A}, N_{B}$ and $N_{a b}$ are known, but this is not always the case. The authors also provide modifications to be carried out in (12) to adapt it to situations where only $N_{A}$ and $N_{B}$ are known or where none of $N_{A}, N_{B}$ or $N_{a b}$ are known. In addition, auxiliary information coming from either one or both frames can be incorporated into the estimation process to improve the accuracy of the estimates. In addition, instead of an analytic form for the variance of this estimator, Rao and $\mathrm{Wu}$ (2010) propose to compute confidence intervals using the bi-section method described by Wu (2005) for one single frame and extending it to the dual frame case. This method constructs intervals of the form $\left\{\theta \mid r_{n s}(\theta)<\chi_{1}^{2}(\alpha)\right\}$, where $\chi_{1}^{2}(\alpha)$ is the $1-\alpha$ quantile from a $\chi^{2}$ distribution with one degree of freedom and $r_{n s}(\theta)$ represents the so called pseudo empirical log likelihood ratio statistic, which can be obtained as a difference of two pseudo empirical likelihood 
functions.

Recently, Ranalli et al. (2013) extended calibration procedures to estimation from dual frame sampling assuming that some kind of auxiliary information is available. For example, assuming there are $p$ auxiliary variables, $\mathbf{x}_{k}\left(x_{1 k}, \ldots, x_{p k}\right)$ is the value taken by such auxiliary variables on unit $k$. Each auxiliary variable may be available only for units in frame $A$, only for units in frame $B$ or for units in the whole population. In addition, it is assumed that the vector of population totals of the auxiliary variables, $\mathbf{t}_{\mathbf{x}}=\sum_{k \in \mathcal{U}} \mathbf{x}_{k}$ is also known. In this context, the dual frame calibration estimator can be defined as follows

$$
\hat{Y}_{C A L D F}=\sum_{k \in S} d_{k}^{C A L D F} y_{k}
$$

where weights $d_{k}^{C A L D F}$ are such that $\min \sum_{k \in s} G\left(d_{k}^{C A L D F}, \breve{d}_{k}\right)$ subject to $\sum_{k \in s} d_{k}^{C A L D F} \mathbf{x}_{k}=\mathbf{t}_{\mathbf{x}}$, with $G(\cdot, \cdot)$ a determined distance measure and

$$
\breve{d}_{k}=\left\{\begin{array}{ll}
d_{k}^{A} & \text { if } k \in a \\
\eta d_{k}^{A} & \text { if } k \in a b \cap s_{A} \\
(1-\eta) d_{k}^{B} & \text { if } k \in a b \cap s_{B} \\
d_{k}^{B} & \text { if } k \in b
\end{array},\right.
$$

where $\eta \in[0,1]$.

Then, with a similar approach to that of $\hat{Y}_{B K A}$, another calibration estimator can be computed as

$$
\hat{Y}_{C A L S F}=\sum_{k \in S} d_{k}^{C A L S F} y_{k}
$$

with weights $d_{k}^{C A L S F}$ verifying that $\min \sum_{k \in S} G\left(d_{k}^{C A L S F}, \tilde{d}_{k}\right)$ subject to $\sum_{k \in s} d_{k}^{C A L S F} \mathbf{x}_{k}=\mathbf{t}_{\mathbf{x}}$, being $\tilde{d}_{k}$ the weights defined in (5).

An estimator of the variance of any calibration estimator can be obtained using Deville's method (Deville, 1993) through the following expression

$$
\hat{V}(\hat{Y})=\frac{1}{1-\sum_{k \in s} a_{k}^{2}} \sum_{k \in s} \frac{d_{k}^{\star}-1}{d_{k}^{\star}}\left(d_{k}^{\star} e_{k}-\sum_{l \in S} a_{l} d_{l}^{\star} e_{l}\right)^{2},
$$

where $d_{k}^{\star}$ is given by (5) or by (14) according to whether we use $\hat{Y}_{C A L S F}$ or $\hat{Y}_{C A L D F}$, respectively. In addition, $a_{k}=\frac{d_{k}^{\star}-1}{d_{k}^{\star}} / \sum_{l \in s} \frac{d_{l}^{\star}-1}{d_{l}^{\star}}$ and $e_{k}$ are the residuals of the generalized regression of $y$ on $\mathbf{x}$.

Some of the estimators described above are particular types of calibration estimators. For example, estimator (8) can be obtained as a particular case of $\hat{Y}_{C A L S F}$ in the case where frame sizes $N_{A}$ and $N_{B}$ are known and the "raking" method is selected for calibration. Having noted this, one can use (16) to calculate an estimator of variance of (8). See Ranalli et al. (2013) for more details.

Table 1 shows a summary of the previous dual frame estimators according to the auxiliary information required. It can be noted that Hartley, FB and BKA estimators can be computed even when no information is available, but they cannot incorporate some auxiliary information when available. PML and SFRR can incorporate information on $N_{A}$ and $N_{B}$, but PEL and CAL type estimators are the most flexible in that they can incorporate any kind of auxiliary information available.

\section{Jackknife variance estimation}

Variance estimation methods exposed so far depend on each specific estimator, so comparisons between variance estimations may lead to incorrect conclusions. Instead, one can consider jackknife, originally proposed by Quenouille $(1949,1956)$ (see Wolter 2007 for a detailed description of this method in survey sampling) and extended to dual frame surveys by Lohr and Rao (2000), which can be used to estimate variances irrespective of the type of estimator allowing us to compare estimated efficiency for different estimators.

For a non-stratified design in each frame, the jackknife estimator of the variance for any of the estimators described, generically denoted by $\hat{Y}_{c}$, is given by

$$
v_{J}\left(\hat{Y}_{c}\right)=\frac{n_{A}-1}{n_{A}} \sum_{i \in s_{A}}\left(\hat{Y}_{c}^{A}(i)-\bar{Y}_{c}^{A}\right)^{2}+\frac{n_{B}-1}{n_{B}} \sum_{j \in s_{B}}\left(\hat{Y}_{c}^{B}(j)-\bar{Y}_{c}^{B}\right)^{2},
$$

with $\hat{Y}_{c}^{A}(i)$ the value of estimator $\hat{Y}_{c}$ after dropping unit $i$ from $s_{A}$ and $\bar{Y}_{c}^{A}$ the mean of values $\hat{Y}_{c}^{A}(i)$. Similarly, one can define $\hat{Y}_{c}^{B}(j)$ and $\bar{Y}_{c}^{B}$.

Jackknife may present an important bias when designs are without replacement. One could, 


\begin{tabular}{lcccc}
\hline & None & $\begin{array}{c}N_{A}, N_{B} \\
\text { known }\end{array}$ & $\begin{array}{c}N_{a}, N_{b} \text { and } \\
N_{a b} \text { known }\end{array}$ & $\begin{array}{c}N_{a}, N_{a b}, N_{b} \text { and } \\
X_{A} \text { and/or } X_{B} \text { known }\end{array}$ \\
\hline Hartley & $\checkmark$ & & & \\
FB & $\checkmark$ & & & \\
PML & & $\checkmark$ & & \\
PEL & $\checkmark$ & $\checkmark$ & $\checkmark$ & $\checkmark$ \\
CalDF & $\checkmark$ & $\checkmark$ & $\checkmark$ & \\
BKA $^{*}$ & $\checkmark$ & & & \\
SFRR $^{*}$ & & $\checkmark$ & & $\checkmark$ \\
CalSF* $^{*}$ & $\checkmark$ & $\checkmark$ & $\checkmark$ & \\
\hline
\end{tabular}

$\left.{ }^{*}\right)$ Inclusion probabilities are known in overlap domain $a b$ for both frames.

Table 1: Estimator's capabilities versus auxiliary information availability

then, incorporate an approximate finite-population correction to estimation to achieve unbiasedness. For example, assuming that a finite-population correction is needed in frame $A$, a modified jackknife estimator of variance, $v_{J}^{*}\left(\hat{Y}_{c}\right)$, can be calculated by replacing $\hat{Y}_{c}^{A}(i)$ in (17) with $\hat{Y}_{c}^{A *}(i)=\hat{Y}_{c}+\sqrt{1-\bar{\pi}_{A}}\left(\hat{Y}_{c}^{A}(i)-\hat{Y}_{c}\right)$, where $\bar{\pi}_{A}=\sum_{k \in s_{A}} \pi_{k}^{A} / n_{A}$.

Consider now a stratified design in each frame, where frame $A$ is divided into $H$ strata and frame $B$ is divided into $L$ strata. From stratum $h$ of frame $A$, a sample of $n_{A h}$ units from the $N_{A h}$ population units in the stratum is drawn. Similarly, in stratum $l$ of frame $B$, one selects $n_{B l}$ units from the $N_{B l}$ composing the stratum. The jackknife estimator of the variance can be defined, then, as follows

$$
v_{J}\left(\hat{Y}_{c}\right)=\sum_{h=1}^{H} \frac{n_{A h}-1}{n_{A h}} \sum_{i \in s_{A h}}\left(\hat{Y}_{c}^{A}(h i)-\bar{Y}_{c}^{A h}\right)^{2}+\sum_{l=1}^{L} \frac{n_{B l}-1}{n_{B l}} \sum_{i \in s_{B l}}\left(\hat{Y}_{c}^{B}(l j)-\bar{Y}_{c}^{B l}\right)^{2},
$$

where $\hat{Y}_{c}^{A}(h i)$ is the value taken by $\hat{Y}_{c}$ after dropping unit $i$ of stratum $h$ from sample $s_{A h}$ and $\bar{Y}_{c}^{A h}$ is the mean of values $\hat{Y}_{c}^{A}(h i) . \hat{Y}_{c}^{B}(l j)$ and $\bar{Y}_{c}^{B l}$ can be defined in a similar way. Again, one can include an approximate finite-population correction in any stratum needing it. In case of a non stratified design in one frame and a stratified design in the other one, previous methods can be combined to obtain the corresponding jackknife estimator of the variance.

Stratified cluster sampling is very common in practice. Now we illustrate the jackknife estimator when a stratified sample of clusters is selected. Suppose that frame $A$ has $H$ strata and stratum $h$ has $N_{A h}$ observation units and $\widetilde{N}_{A h}$ primary sampling units (clusters), of which $\widetilde{n}_{A h}$ are sampled. Frame $B$ has $L$ strata, and stratum $l$ has $N_{B l}$ observation units and $\widetilde{N}_{B h}$ primary sampling units, of which $\widetilde{n}_{B l}$ are sampled.

To define the jackknife estimator of the variance, let $\widetilde{Y}_{c}^{A}(h j)$ be the estimator of the same form as $\hat{Y}_{c}$ when the observations of sample primary sampling unit $j$ of stratum $h$ from sample in frame $A$ are omitted. Similarly, $\widetilde{Y}_{c}^{B}(l k)$ is of the same form as $\hat{Y}_{c}$ when the observations of sample primary sampling unit $k$ of stratum $l$ from sample in frame $B$ are omitted. The jackknife variance estimator is then given by:

$$
v_{J}\left(\hat{Y}_{c}\right)=\sum_{h=1}^{H} \frac{\widetilde{n} A h-1}{\widetilde{n}_{A h}} \sum_{j=1}^{\widetilde{n}_{A h}}\left(\widetilde{Y}_{c}^{A}(h j)-\widetilde{Y}_{c}^{A h}\right)^{2}+\sum_{l=1}^{L} \frac{\widetilde{n}_{B l}-1}{\widetilde{n}_{B l}} \sum_{k \in s_{B l}}\left(\widetilde{Y}_{c}^{B}(l k)-\widetilde{Y}_{c}^{B l}\right)^{2},
$$

where $\widetilde{Y}_{c}^{A h}$ is the mean of values $\widetilde{Y}_{c}^{A}(h j)$ and $\widetilde{Y}_{c}^{B l}$ is the mean of values $\widetilde{Y}_{c}^{B}(l k)$.

\section{The $R$ package Frames2}

Frames2 is a new $\mathrm{R}$ package for point and interval estimation from dual frame sampling. It consists of eight main functions (Hartley, FB, BKA, SFRR, PML, PEL, CalSF and CalDF), each of them implementing one of the estimators described in the previous sections. The package also includes an additional function called Compare which provides a summary with all possible estimators that can be computed from the information provided as input. Moreover, six extra functions implementing auxiliary operations, like computation of Horvitz-Thompson estimators or of the covariance between two 


\begin{tabular}{|c|c|c|c|}
\hline & user & system & elapsed \\
\hline & \multicolumn{3}{|c|}{$n_{A}=10605, n_{B}=13635$} \\
\hline Hartley & 0.01 & 0.02 & 0.04 \\
\hline FB & 0.05 & $<0.01$ & 0.07 \\
\hline BKA & 0.03 & $<0.01$ & 0.05 \\
\hline PML & 0.02 & 0.02 & 0.03 \\
\hline SFRR & 0.03 & 0.03 & 0.07 \\
\hline CalSF & 0.03 & $<0.01$ & 0.06 \\
\hline \multirow[t]{2}{*}{ CalDF } & 0.04 & 0.01 & 0.05 \\
\hline & \multicolumn{3}{|c|}{$n_{A}=105105, n_{B}=135135$} \\
\hline Hartley & 0.11 & 0.06 & 0.19 \\
\hline FB & 0.27 & 0.07 & 0.32 \\
\hline BKA & 0.13 & 0.05 & 0.17 \\
\hline PML & 0.16 & 0.02 & 0.18 \\
\hline SFRR & 0.42 & 0.12 & 0.54 \\
\hline CalSF & 0.20 & 0.08 & 0.30 \\
\hline CalDF & 0.22 & 0.07 & 0.31 \\
\hline
\end{tabular}

Table 2: User, system and elapsed times (in seconds) for estimators considering different sample sizes.

Horvitz-Thompson estimators, have also been included in the package to achieve a more understandable code. Finally, the package includes eight more functions, one for each estimator, for the calculation of confidence intervals based on the jackknife variance estimator.

A notable feature of these functions is the strong argument check. Functions check general aspects such as the presence of NA or NaN values in its arguments, the number of main variables considered in the frames (that should match), the length of the arguments in each frame (that should also match) and the values for arguments indicating the domain each unit belongs to (which only can be "a" or " $a b$ " for frame $A$ or " $b$ " or "ba" for frame B). If any issue is encountered, the function displays an error message indicating what the problem is and what argument causes it, so that the user can manage errors easily. Furthermore, each function has additional checks depending on its specific characteristics or arguments. The main aim of this exhaustive check is to guarantee validity of the arguments, so one can avoid, to the extent possible, issues during computation.

Much attention has also been devoted to computational efficiency. Frequently, populations in a survey are extremely large or it is needed to keep sampling error below a certain value. As a consequence, one needs to consider large sample sizes, often in the order of tens of thousands sampling units. In these situations, computational efficiency of functions is essential, particularly when several variables are considered. Otherwise, the user can face high runtimes and heavy computational loads. In this sense, functions of Frames 2 are developed according to strict efficiency measures, using the power of $\mathrm{R}$ to use matrix calculation to avoid loops and increase the computational efficiency.

Table 2 shows user and system times necessary to compute estimators using an Intel(R) Core(TM) i7-3770 at $3.40 \mathrm{GHz}$ when different sample sizes are considered. Elapsed time is also included to get an idea about the real time user needs to get estimations.

Functions of Frames2 have been implemented from an user-oriented perspective to increase usability. In this sense, most input parameters (which are the communication channel between the user and the function) are divided into two groups, depending on the frame they come from. This is to adapt functions as much as possible to the usual estimation procedure, in which the first step is to draw two independent samples, one from each frame. On the other hand, estimation details are managed internally by functions so that they are not visible for the user, who does not need to manage them.

Construction of functions has been carried out so that they perform properly in as many situations as possible. As noted in the introductory section, one can face several situations when using two 
sampling frames depending on their relative positions. Although the most common is the one depicted in Figure 1, cases shown in Figures 2 and 3 may arise as well. All estimators described except PEL can be modified to cover these three situations, so corresponding functions of Frames 2 include necessary changes to produce estimates irrespective of the situation.

On the other hand, it is usual, when conducting a survey, to collect information on many variables of interest. To adapt to such situations, all functions are programmed to produce estimates when there are more than one variable of interest with only one call. To this end, parameters containing information about main variables observed in each frame can be either vectors, when only one variable is considered or matrices or data frames, when there are several variables under study. Cases in which the main aim of the survey is the estimation of population means or proportions are also very frequent. Hence, from the estimation of the population total for a variable, functions compute estimation of the mean as $\hat{Y}=\hat{Y} / \hat{N}$. To obtain the estimation of the population size, functions internally apply the estimation procedure at issue to indicator vectors $\mathbf{1}_{A}$ and $\mathbf{1}_{B}$ of sizes $n_{A}$ and $n_{B}$, respectively.

To get maximum flexibility, functions have been programmed to calculate estimates in cases in which the user disposes of first and second order inclusion probabilities and in those other in which only first order ones are available, indistinctly. Knowledge of both first and second order inclusion probabilities is a strong assumption that does not always occur in practice. However, when calculating most of the estimators described in previous sections, second order inclusion probabilities are needed in many steps of the estimation procedure, mainly in computing estimated variances of a HorvitzThompson estimator or estimated covariances between two Horvitz-Thompson estimators. As an alternative, one can obtain variance estimations from only first order inclusion probabilities applying Deville's method reported in (16), by substituting residuals $e_{k}$ with the values of the variable of interest, $y_{k}$. Covariance estimations are also obtained from variances through the following expression

$$
\widehat{\operatorname{Cov}}(\hat{Y}, \hat{X})=\frac{\hat{V}(\hat{Y}+\hat{X})-\hat{V}(\hat{Y})-\hat{V}(\hat{X})}{2} .
$$

To cover both cases, the user has the possibility to consider different data structures for parameters relating to inclusion probabilities. So, if both first and second order inclusion probabilities are available, these parameters will be square matrices, whereas if only first order inclusion probabilities are known, these arguments will be vectors. The only restriction here is that the type of both should match.

As can be deduced from previous sections, an essential aspect when computing estimates in dual frames is to know the domain each unit belongs to. Character vectors domains_A and domains_B are used for this purpose. The former can take values "a" or "ab", while the latter can take values " $b$ " or "ba". Any other value will be considered as incorrect.

\section{Data description}

To illustrate how functions operate, we use the data sets DatA and DatB, both included in the package. DatA contains information about $n_{A}=105$ households selected through a stratified sampling design from the $N_{A}=1735$ households forming frame $A$. More specifically, frame A has been divided into 6 strata of sizes $N_{h A}=(727,375,113,186,115,219)$ from which simple random without replacement samples of sizes $n_{h A}=(15,20,15,20,15,20)$ have been drawn. On the other hand, a simple random without replacement sample of $n_{B}=135$ households has been selected from the $N_{B}=1191$ households in frame $B$. The size of the overlap domain for this case is $N_{a b}=601$. This situation is depicted in Figure 4.

Both data sets contain information about the same variables. To better understand their structure, we report the first three rows of DatA:

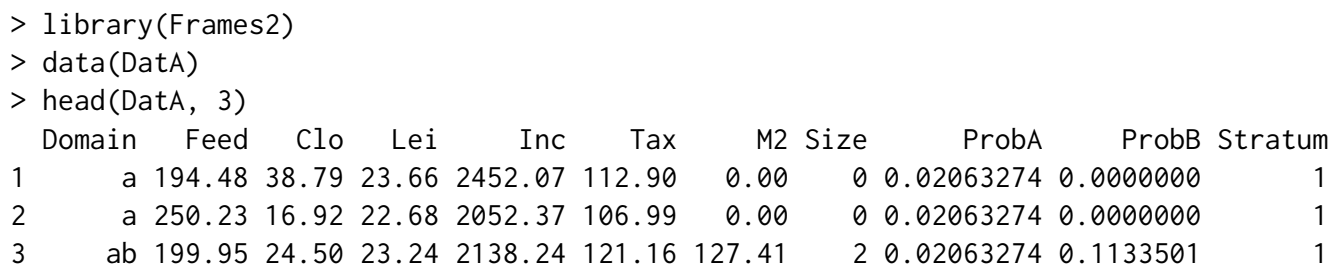

Each data set incorporates information about three main variables: Feeding, Clothing and Leisure. Additionally, there are two auxiliary variables for the units in frame $A$ (Income and Taxes) and another two variables for units in frame $B$ (Metres2 and Size). Corresponding totals for these auxiliary variables are assumed known in the entire frame and they are $T_{I n c}^{A}=4300260, T_{\text {Tax }}^{A}=215577, T_{M 2}^{B}=176553$ and $T_{\text {Size }}^{B}=3529$. Finally, a variable indicating the domain each unit belongs to and two variables showing the first order inclusion probabilities for each frame complete the data sets. 


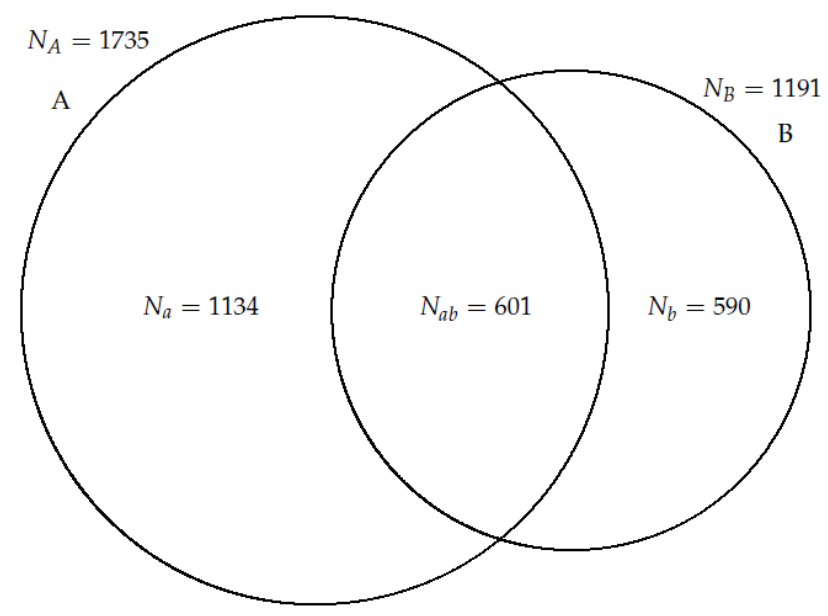

Figure 4: Frame and domain sizes for the data sets.

Numerical square matrices PiklA and PiklB, with dimensions $n_{A}=105$ and $n_{B}=135$, are also used as probability inclusion matrices. These matrices contains second order inclusion probabilities and first order inclusion probabilities as diagonal elements. To check the appearance of these matrices the first submatrix of order 6 of PiklA is shown.

$>\operatorname{data}(\mathrm{PiklA})$

$>\operatorname{PiklA}[1: 6,1: 6]$

$$
[, 1]
$$

[,4]

$[, 5]$

$[, 6]$

$\left[\begin{array}{llllllllll}{[1,]} & 0.020632737 & 0.000397876 & 0.000397876 & 0.000397876 & 0.000397876 & 0.000397876\end{array}\right.$

$[2] \quad 0.000397876 \quad 0.020632737 \quad 0.000397876 \quad 0.000397876 \quad 0.000397876 \quad$,

$\left[\begin{array}{lllllllll}3,] & 0.000397876 & 0.000397876 & 0.020632737 & 0.000397876 & 0.000397876 & 0.000397876\end{array}\right.$

$\left[\begin{array}{lllllllllll} & 0.000397876 & 0.000397876 & 0.000397876 & 0.020632737 & 0.000397876 & 0.000397876\end{array}\right.$

$\left[\begin{array}{lllllllll}5,] & 0.000397876 & 0.000397876 & 0.000397876 & 0.000397876 & 0.020632737 & 0.000397876\end{array}\right.$

$\left[\begin{array}{llllllllll}6,] & 0.000397876 & 0.000397876 & 0.000397876 & 0.000397876 & 0.000397876 & 0.020632737\end{array}\right.$

\section{No auxiliary information}

When there is no further information than the one on the variables of interest, one can calculate some of the estimators described in previous sections (as, for example, (1) or (3)) as follows

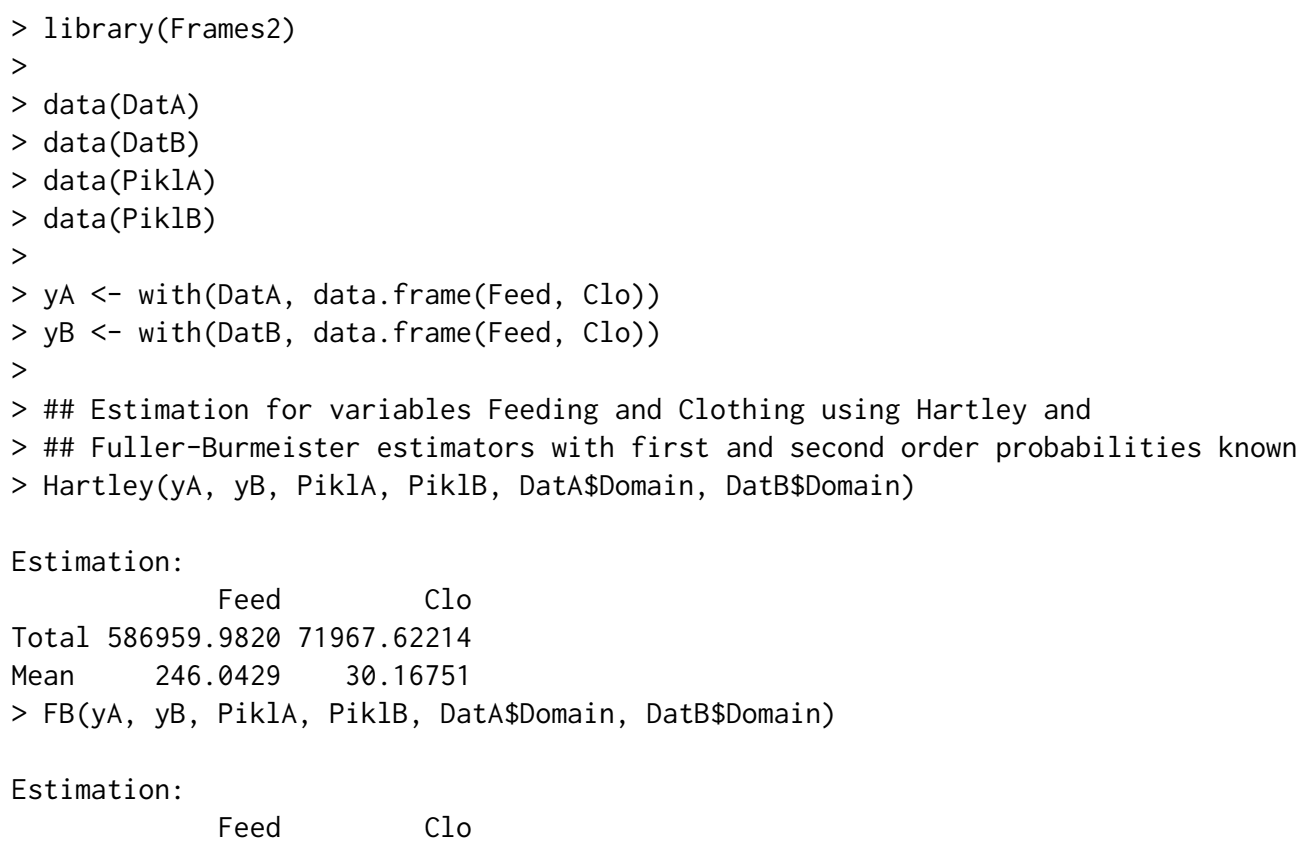




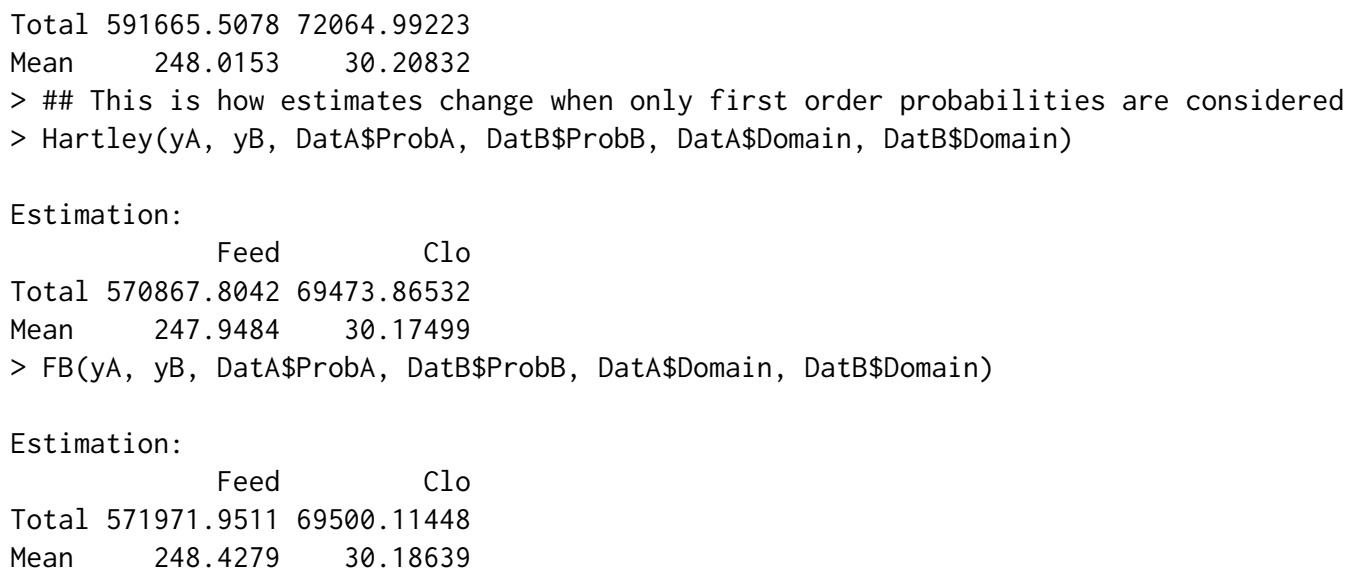

As the result, an object of class "EstimatorDF" is returned, showing, by default, estimations for the population total and mean for the two considered variables. In general, $m$ columns will be displayed, one for each of the $m$ variables estimated. Further information about the estimation process (as variance estimations or values of parameters involved in estimation) can be displayed by using function summary.

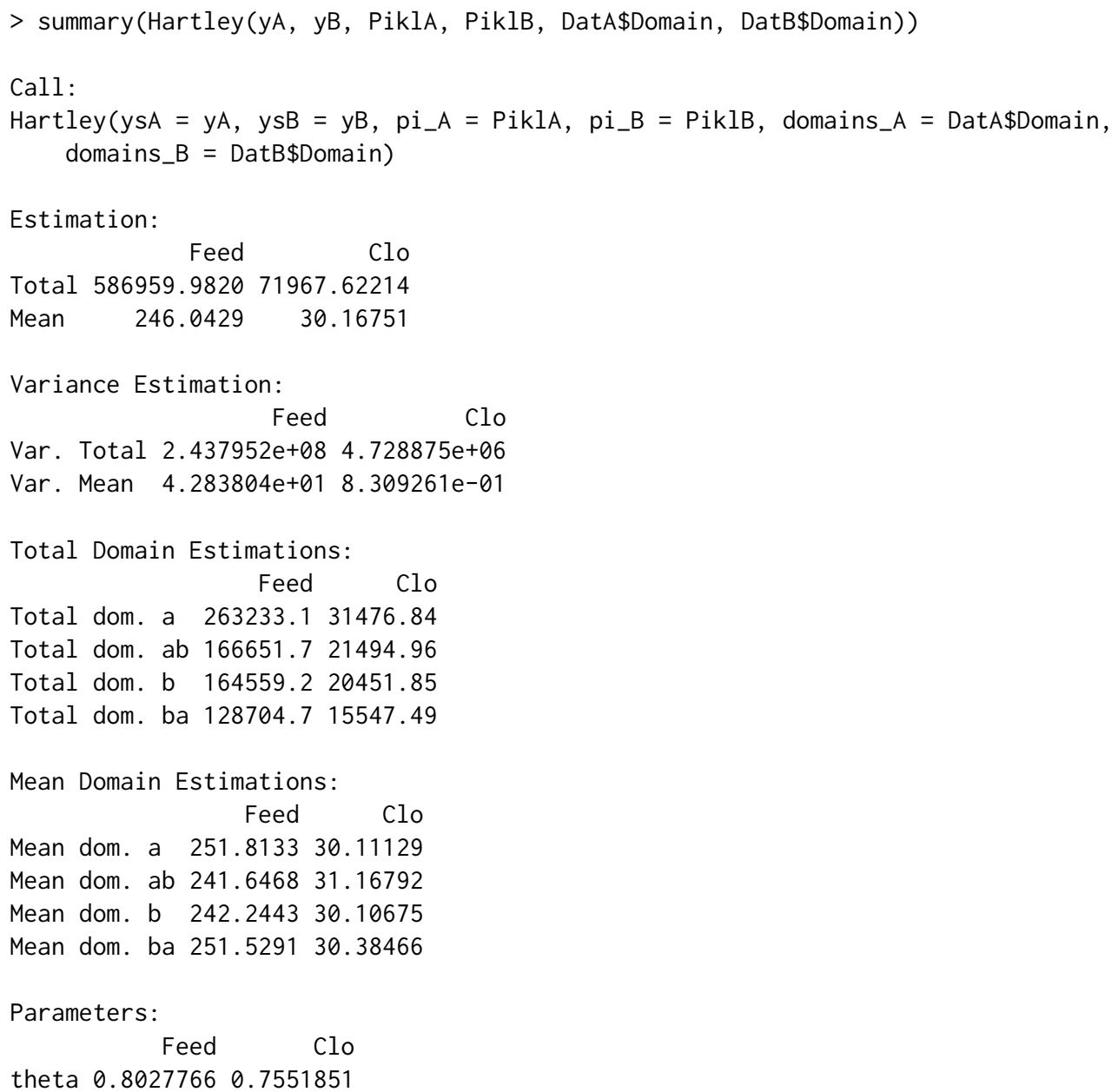

The previous output shows in the component Estimation the estimations of the population total and the population mean computed using the Harley estimator, that is, $\hat{Y}_{H}$ and $\hat{\bar{Y}}_{H}$. Estimated variances of these estimations, $\hat{V}\left(\hat{Y}_{H}\right)$ and $\hat{V}\left(\hat{\bar{Y}}_{H}\right)$, are displayed in component Variance Estimation. In the section Total Domain Estimations we can see estimations $\hat{Y}_{a}, \hat{Y}_{a b}^{A}, \hat{Y}_{b}$ and $\hat{Y}_{a b}^{B}$. Estimates for the population mean for each domain, $\hat{\bar{Y}}_{a}, \hat{\bar{Y}}_{a b}^{A}, \hat{\bar{Y}}_{b}$ and $\hat{\bar{Y}}_{a b}^{B}$ are displayed in the component Mean Domain Estimations. Finally, $\hat{\theta}$, the estimated value of the parameter involved in computation of the Hartley estimator is shown. 
This additional information depends on the way each estimator is formulated. Thus, for example, extra information will include a parameter component when applied to a call to the Fuller-Burmeister estimator (and values of estimates for $\beta_{1}$ and $\beta_{2}$ will be displayed there), but not when applied to a call to the Bankier-Kalton-Anderson estimator (because no parameters are used when computing this estimator).

Results slightly change when a confidence interval is required. In that case, the user has to indicate the confidence level desired for the interval through argument conf_level (default is NULL) and add it to the list of input parameters. The function calculates, then, a confidence interval based on the pivotal method. This method yields a confidence interval as follows: $\hat{Y} \pm z_{\alpha / 2} \sqrt{\hat{V}(\hat{Y})}$ where $z_{\alpha / 2}$ is the critical value of a standard normal distribution. Only for the case of PEL, confidence intervals are based on a $\chi^{2}$ distribution and the bi-section method (Rao and $\left.W u, 2010\right)$. In this case, default output will show 6 rows for each variable, lower and upper boundaries for confidence intervals are displayed together with estimates. So, one can obtain a $95 \%$ confidence interval for estimations in the last two of the previous four cases in this way.

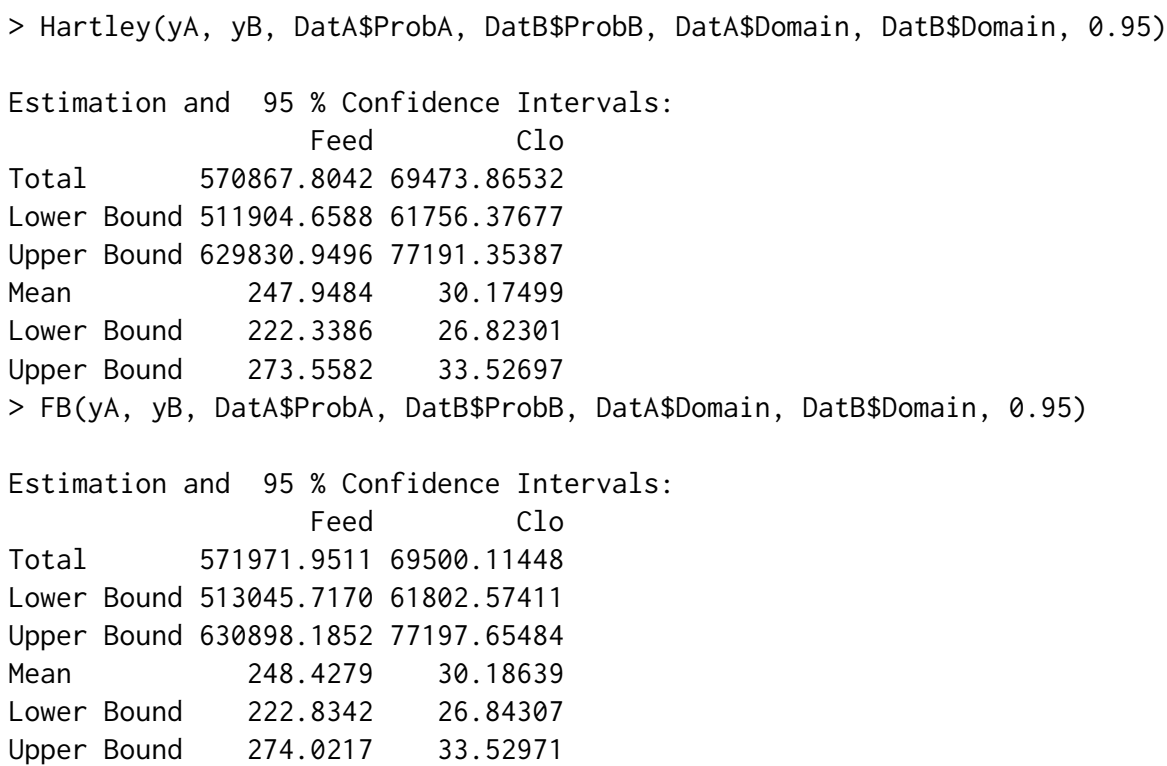

For estimators constructed as (6), numeric vectors pik_ab_B and pik_ba_A of lengths $n_{A}$ and $n_{B}$ should be added as arguments. While pik_ab_B represents first order inclusion probabilities according to sampling design in frame $B$ for units belonging to the overlap domain selected in the sample drawn from frame $A$, pik_ba_A contains first order inclusion probabilities according to the sampling design in frame $A$ for units belonging to the overlap domain selected in sample drawn from frame $B$.

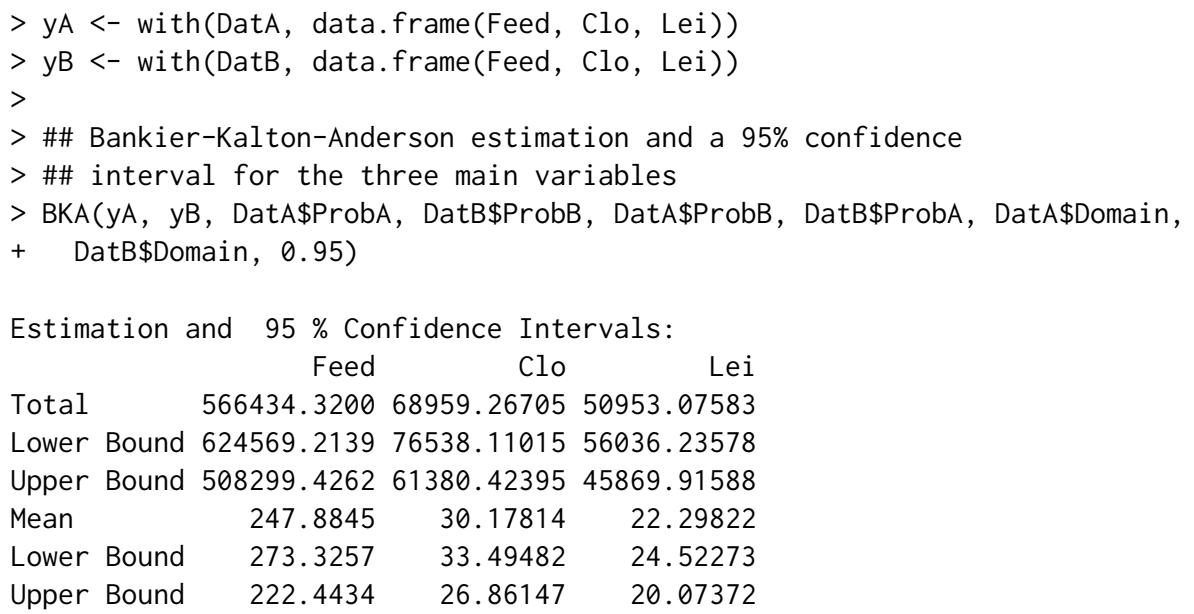

Note that these examples include just a few of the estimators that can be used when no auxiliary information is known. As noted in Table 1, other estimators, as those in (12), (13) or (15), can be also calculated in this case. In this context, function Compare is quite useful, since it returns all possible estimators that can be computed according to the information provided as input.

> Compare(yA, yB, DatA\$ProbA, DatB\$ProbB, DatA\$Domain, DatB\$Domain)

\$Hartley 
Estimation:

\begin{tabular}{|c|c|c|c|}
\hline & Feed & $\mathrm{Clo}$ & Lei \\
\hline Total & 570867.8042 & 69473.865325 & 51284.2727 \\
\hline Mean & 247.9484 & 30.17499 & 22.2746 \\
\hline \multicolumn{4}{|c|}{ \$FullerBurmeister } \\
\hline \multicolumn{4}{|c|}{ Estimation: } \\
\hline & Feed & $\mathrm{Clo}$ & Lei \\
\hline Total & 571971.9511 & 69500.114485 & 51210.03819 \\
\hline Mean & 248.4279 & 30.18639 & 22.24236 \\
\hline \multicolumn{4}{|l|}{ \$PEL } \\
\hline \multicolumn{4}{|c|}{ Estimation: } \\
\hline & Feed & $\mathrm{Clo}$ & Lei \\
\hline Total & $1.791588 \mathrm{e}+08$ & $2.663164 \mathrm{e}+06$ & $51.455533 e+06$ \\
\hline Mean & $2.479314 \mathrm{e}+02$ & $2.011373 e+01$ & $2.235969 \mathrm{e}+01$ \\
\hline \multicolumn{4}{|c|}{ \$Calibration_DF } \\
\hline \multicolumn{4}{|c|}{ Estimation: } \\
\hline & Feed & Clo & Lei \\
\hline Total & 595162.2604 & 72214.133515 & 53108.5059 \\
\hline Mean & 248.8422 & 30.19332 & 22.2051 \\
\hline
\end{tabular}

Using appropriate indicator variables as variables of interest, one can also estimate the overlap domain size, as shown below:

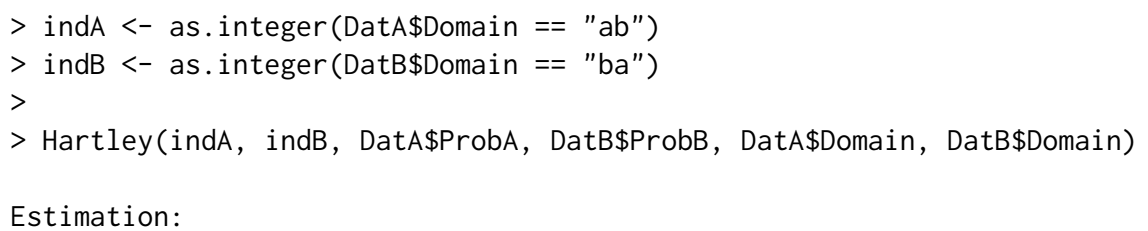

Estimation:

Total 534.2743208

Mean 0.2320545

$>$ BKA(indA, indB, DatA\$ProbA, DatB\$ProbB, DatA\$ProbB, DatB\$ProbA, DatA\$Domain,

+ DatB $\$$ Domain)

Estimation:

Total 560.4121771

Mean $\quad 0.2452491$

\section{Auxiliary information about frame sizes}

For estimators requiring frame sizes known, as (8) or (9), it is needed to incorporate two additional input arguments, N_A and N_B. There is also a group of estimators, including (12) and (15), that, even though able to provide estimations without the need of auxiliary information, can use frame sizes to improve their precision. The following examples show the performance of these estimators.

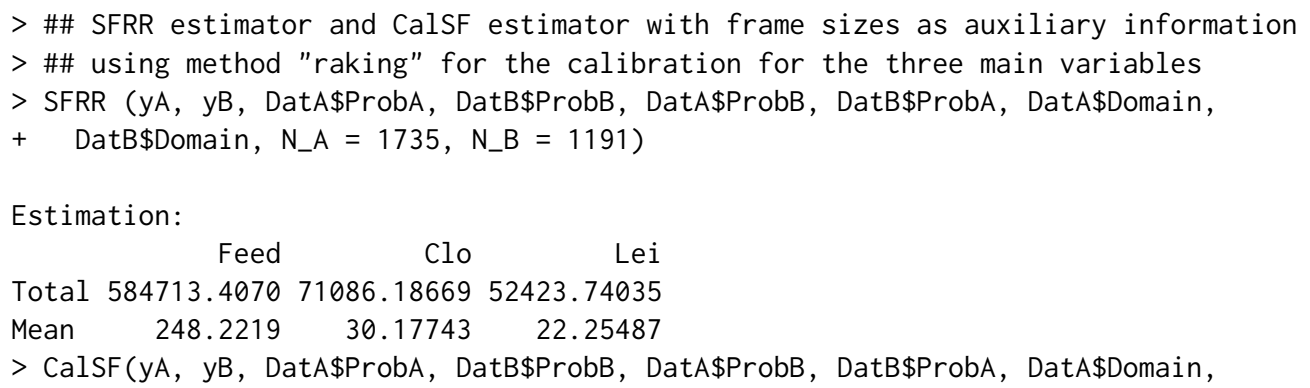


+ DatB\$Domain, N_A $=1735, \quad$ N_B $=1191$, met $=$ "raking")

Estimation:

Feed Clo Lei

Total 584713.4070 71086.18669 52423.74035

Mean $\quad 248.2219 \quad 30.17743 \quad 22.25487$

As highlighted previously, both results match. Note that the argument met of the SF calibration estimator indicates the method used in the calibration procedure. The possibility of choosing the calibration method is given by the fact that computation of both SF and DF calibration estimators is based on the function calib from package sampling (Tillé and Matei, 2012), which can manage three different calibration methods, each one associated with one particular distance measure. These methods are: linear, raking and logit.

The requirement of knowing probabilities of inclusion in both frames for the units in the overlap domain may be restrictive in some cases. As an alternative, in cases where frame sizes are known but this condition is not met, it is possible to calculate dual frame estimators as (9), (12) or (13). Next, it is illustrated how to obtain some of these estimators with Frames2.

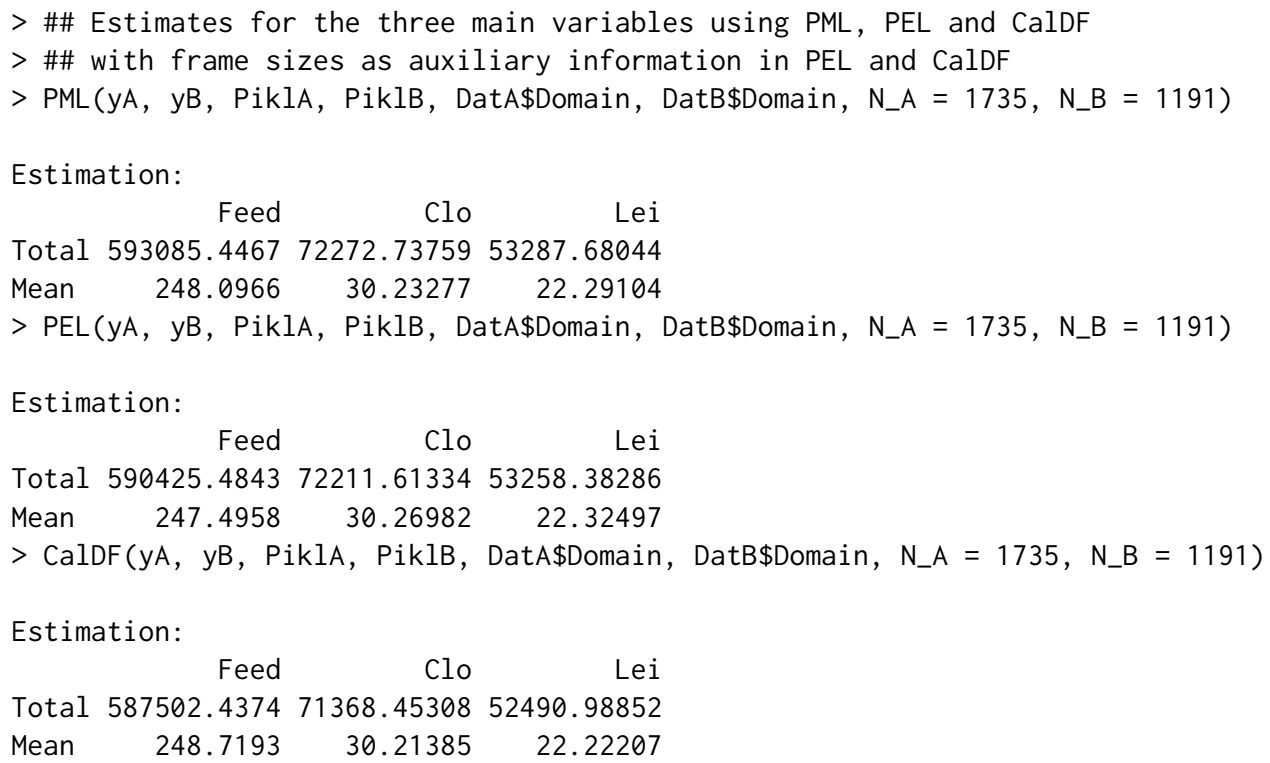

To calculate the PEL estimator, computational algorithms for the pseudo empirical likelihood method for the analysis of complex survey data presented by $\mathrm{Wu}$ (2005) are used.

\section{Auxiliary information about domain sizes}

In addition to the frame sizes, in some cases, it is possible to know the size of the overlap domain, $N_{a b}$. Generally, this considerably improves the precision of the estimates. This situation has been taken into account when constructing functions implementing estimators (12), (13) and (15), so the user can incorporate this information through parameter N_ab, as shown below

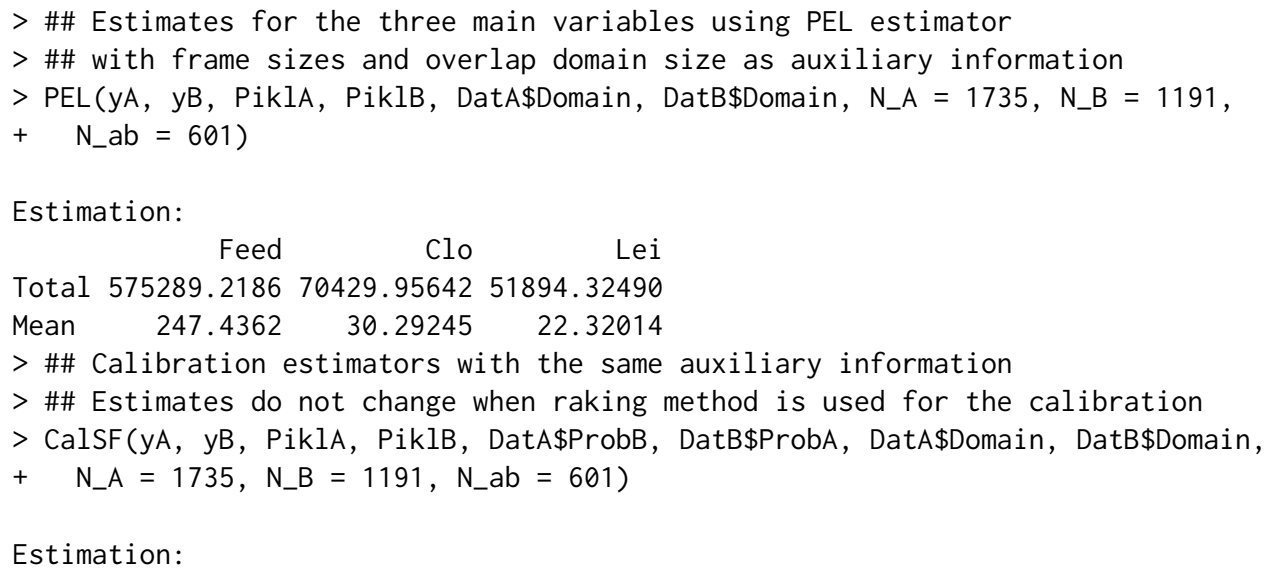




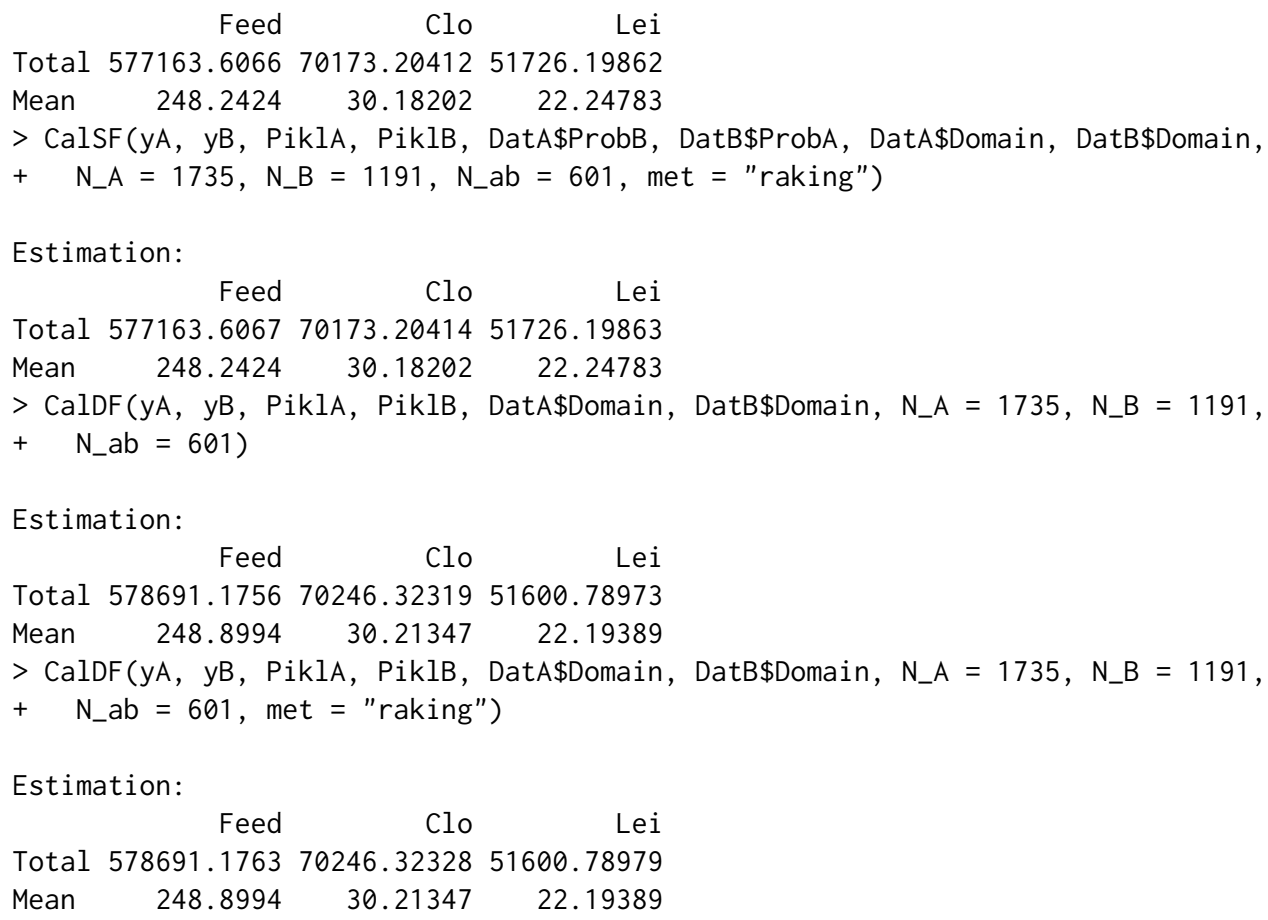

Note that in this case, calibration estimators provide the same results irrespective of the distance function employed. This is an interesting property that calibration estimators show only in the case in which all the domain sizes are known and used for calibration (see Deville 1993).

\section{Auxiliary information about additional variables}

On the other hand, some of the estimators are defined such that they can incorporate auxiliary information into the estimation process. This is the case for estimators (12), (13) and (15). Functions implementing them are also able to manage auxiliary information. To achieve maximum flexibility, functions implementing estimators (12), (13) and (15) are prepared to deal with auxiliary information when it is available only in frame $A$, only in frame $B$ or in both frames. For instance, auxiliary information collected from frame $A$ should be incorporated into functions through three arguments: XSAFrameA and XsBFrameA, numeric vectors, matrices or data frames (depending on the number of auxiliary variables in the frame); and XA, a numeric value or vector of length indicating population totals for the auxiliary variables considered in frame $A$. Similarly, auxiliary information in frame $B$ is incorporated into each function through arguments XsAFrameB, xsBFrameB and XB. If auxiliary information is available in the whole population, this must be indicated through parameters $x \mathrm{ST}$ and $X$. In the following example, one can see how to calculate estimators using different type of auxiliary information

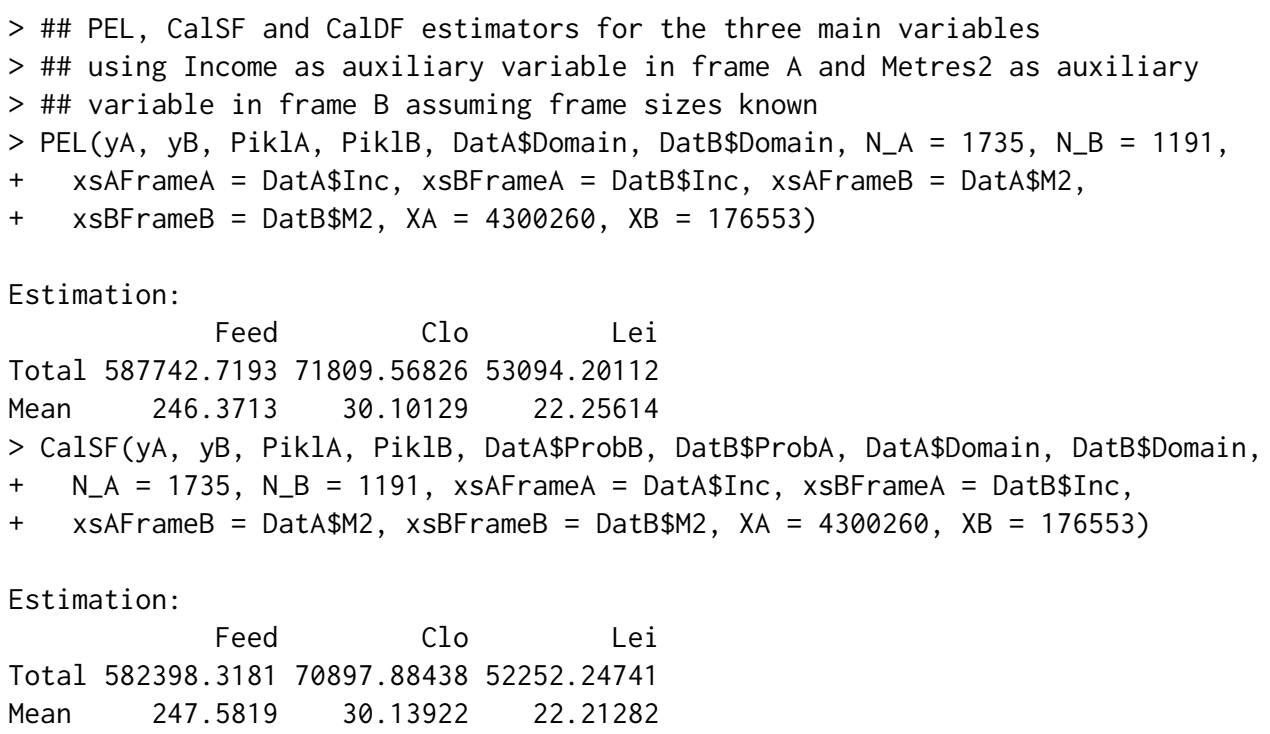


$>\operatorname{CalDF}(y A, y B, P i k l A, P i k l B$, DatA\$Domain, DatB\$Domain, N_A = 1735, N_B = 1191,

$+\quad x s A F r a m e A=$ DatA $\$$ Inc, $x$ sBFrameA $=$ DatB $\$$ Inc,$x s A F r a m e B=$ DatA $\$ M 2$,

$+\quad x$ sBFrameB $=$ DatB\$M2, $X A=4300260, X B=176553$ )

Estimation:

Feed Clo Lei

Total $585185.4497 \quad 71194.6114852346 .43878$

Mean $\quad 247.8075 \quad 30.14866 \quad 22.16705$

$>$ \#\# Now, assume that overlap domain size is also known

$>$ PEL (yA, yB, PiklA, PiklB, DatA\$Domain, DatB\$Domain, N_A $=1735$, N_B $=1191$,

$+\quad N_{-} a b=601, x s A F r a m e A=$ DatA $\$ I n c, x s B F r a m e A=$ DatB $\$ I n c$,

$+\quad$ xsAFrameB $=$ DatA\$M2, $x$ sBFrameB $=$ DatB $\$ 2, \quad X A=4300260, \quad X B=176553)$

Estimation:

Feed Clo Lei

Total 572611.699769991 .7480351737 .56089

Mean $\quad 246.2846 \quad 30.10398 \quad 22.25271$

$>$ CalSF (yA, yB, PiklA, PiklB, DatA\$ProbB, DatB\$ProbA, DatA\$Domain, DatB\$Domain,

$+\quad$ N_A $=1735, \quad$ N_B $=1191, \quad N_{-} a b=601, x$ sAFrameA = DatA $\$$ Inc, $x$ sBFrameA = DatB $\$$ Inc ,

$+\quad \mathrm{xsAFrameB}=$ DatA\$M2, $\mathrm{xsBFrameB}=$ DatB\$M2, $X A=4300260, \quad \mathrm{XB}=176553$ )

Estimation:

Feed Clo Lei

Total 575636.787670076 .7848551628 .27583

Mean $\quad 247.5857 \quad 30.14055 \quad 22.20571$

$>\operatorname{CalDF}(y A, y B, P i k l A, P i k l B$, DatA\$Domain, DatB\$Domain, N_A $=1735, \quad$ N_B $=1191$,

$+\quad N_{-} a b=601, x$ SAFrameA $=$ DatA $\$$ Inc, $x$ sBFrameA $=$ DatB $\$ I n c$,

$+\quad x$ sAFrameB $=$ DatA $\$ 22, \quad x s B F r a m e B=$ DatB $\$ M 2, \quad X A=4300260, X B=176553)$

Estimation:

Feed Clo Lei

Total 576630.7609 70102.003751477 .16737

Mean $\quad 248.0132 \quad 30.1514 \quad 22.14072$

\section{Interval estimation based on jackknife variance estimation}

Finally, eight additional functions have been included, each of them calculating confidence intervals based on jackknife variance estimation for each estimator. To carry out variance estimation using the jackknife method, in addition to parameters to calculate each specific estimator, the user has to indicate through arguments $s d A$ and $s d B$ the sampling design applied in each frame. Possible values are "srs" (simple random sampling without replacement), "str" (stratified sampling), "pps" (probabilities proportional to size sampling), "clu" (cluster sampling) or "strclu" (stratified cluster sampling). Default is "srs" for both frames. If a stratified or a cluster sampling has been carried out in one of the frames, it is needed to include information about the strata or the clusters. Furthermore, the user is able to include a finite population correction factor in each frame by setting to TRUE the parameters $f c p A$ and $f c p B$, set by default to FALSE. As the main purpose of the functions is to obtain confidence intervals, parameter conf_level is now mandatory. As noted, these functions can be used, for example, to make comparisons between efficiency of estimators, as shown in the next example.

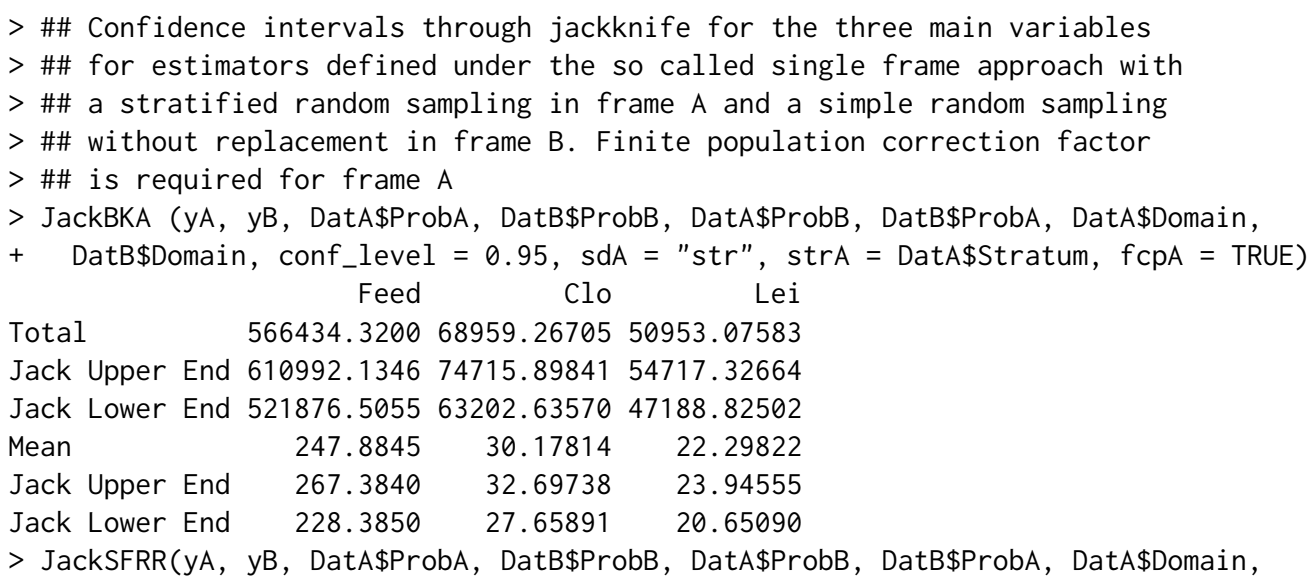


+ DatB\$Domain, N_A $=1735$, N_B $=1191$, conf_level $=0.95$, sdA $=$ "str",

$+\operatorname{str} A=$ DatA\$Stratum, $f(p A=$ TRUE)

Feed Clo Lei

Total 584713.4070 71086.1866952423 .74035

Jack Upper End 619959.0338 76576.74587 55204.67760

Jack Lower End 549467.7802 65595.62751 49642.80309

$\begin{array}{llll}\text { Mean } & 248.2219 & 30.17743 & 22.25487\end{array}$

Jack Upper End $263.1843 \quad 32.50828 \quad 23.43543$

Jack Lower End $233.2595 \quad 27.84659 \quad 21.07431$

$>$ JackCalSF (yA, yB, DatA\$ProbA, DatB\$ProbB, DatA\$ProbB, DatB\$ProbA, DatA\$Domain,

+ DatB $\$$ Domain, N_A $=1735, \quad$ N_B $=1191, N_{-} a b=601$, conf_level $=0.95$, sdA = "str",

$+\quad$ strA $=$ DatA\$Stratum, $f c p A=$ TRUE)

Feed Clo Lei

Total 577163.6066 70173.20412 51726.19862

Jack Upper End 599105.4275 73516.53187 53165.97439

Jack Lower End 555221.7858 66829.87636 50286.42285

$\begin{array}{llll}\text { Mean } & 248.2424 & 30.18202 & 22.24783\end{array}$

Jack Upper End $\quad 257.6798 \quad 31.62001 \quad 22.86709$

Jack Lower End $238.8051 \quad 28.74403 \quad 21.62857$

$>$ \#\# Same for a selection of dual frame estimators

> JackHartley (yA, yB, DatA\$ProbA, DatB\$ProbB, DatA\$Domain, DatB\$Domain,

+ conf_level $=0.95$, sdA $=$ "str", strA = DatA\$Stratum, fcpA $=$ TRUE)

Feed Clo Lei

Total $\quad 570867.8042 \quad 69473.86532 \quad 51284.27265$

Jack Upper End 610664.7131 74907.33129 54782.33083

Jack Lower End 531070.8954 64040.39934 47786.21447

$\begin{array}{llll}\text { Mean } & 247.9484 & 30.17499 & 22.27460\end{array}$

Jack Upper End $265.2336 \quad 32.53494 \quad 23.79393$

Jack Lower End $230.6631 \quad 27.81504 \quad 20.75527$

$>$ JackPML (yA, yB, DatA\$ProbA, DatB\$ProbB, DatA\$Domain, DatB\$Domain,

$+\quad$ N_A $=1735$, N_B $=1191$, conf_level $=0.95$, sdA $=$ "str", $\operatorname{str} A=$ DatA $\$$ Stratum,

$+\quad$ f $P$ A $=$ TRUE)

Feed Clo Lei

Total $\quad 594400.632072430 .0583453408 .30337$

Jack Upper End 626443.7529 76885.06491 56003.77592

Jack Lower End 562357.511167975 .0517650812 .83082

$\begin{array}{llll}\text { Mean } & 248.0934 & 30.23115 & 22.29178\end{array}$

Jack Upper End $261.4677 \quad 32.09060 \quad 23.37509$

Jack Lower End $234.7191 \quad 28.37171 \quad 21.20847$

$>$ JackCalDF (yA, yB, DatA\$ProbA, DatB\$ProbB, DatA\$Domain, DatB\$Domain, N_A $=1735$,

$+\quad \mathrm{N} \_B=1191, \quad \mathrm{~N} \_a b=601$, conf_level $=0.95$, sdA $=$ "str", strA $=$ DatA\$Stratum,

$+\quad \mathrm{fCPA}=\mathrm{TRUE})$

Total $\quad 578895.696170230 .1130651570 .55683$

Jack Upper End 601626.7000 73614.66702 53037.42260

Jack Lower End 556164.6921 66845.55910 50103.69107

$\begin{array}{lll}\text { Mean } \quad 248.9874 & 30.20650 & 22.18088\end{array}$

Jack Upper End $258.7642 \quad 31.66222 \quad 22.81179$

Jack Lower End $\quad 239.2106 \quad 28.75078 \quad 21.54997$

\section{An application to a real telephone survey}

In the example above data are separated into two data sets DatA and DatB containing domain information. But in practice, it is common to have a joint data set including units from both samples in which there is not a specific variable indicating the domain where each individual is placed. However, we can easily split the dataset and format it, so functions of Frames 2 can be applied. To illustrate how to do this, we are going to use dataset Dat, which includes some of the variables collected in a real dual frame survey.

Data included in Dat comes from an opinion survey on the Andalusian population with respect to immigration. This survey is conducted using telephone interviews on adults using two sampling frames: one for landlines and another one for cell phones. From the landline frame, a stratified sample of size 1919 was drawn, while from the cell phone frame, a sample of size 483 is drawn using simple random sampling without replacement. First-order inclusion probabilities were computed from a 
stratified random design in the landline frame and modified taking into account the number of fixed lines and adults in the household. In the cell phone frame first-order inclusion probabilities were computed and modified, given the number of cell phone numbers per individual. At the time of data collection, frame sizes of land and cell phones were 4,982,920 and 5,707,655, respectively, and the total population size was 6,350,916.

The data set includes information about 7 variables: Drawnby, which takes value 1 if the unit comes from the landline sample and value 2 if it comes from the cell phone sample; Stratum, which indicates the stratum each unit belongs to (for individuals in the cell phone frame, value of this variable is NA); Opinion the response to the question: "Do you think that immigrants currently living in Andalusia are quite a lot?" with value 1 representing "yes" and value 0 representing "no"; Landl ine and Cell, which record whether the unit possess a landline or a cell phone, respectively. First order inclusion probabilities are also included in the data set.

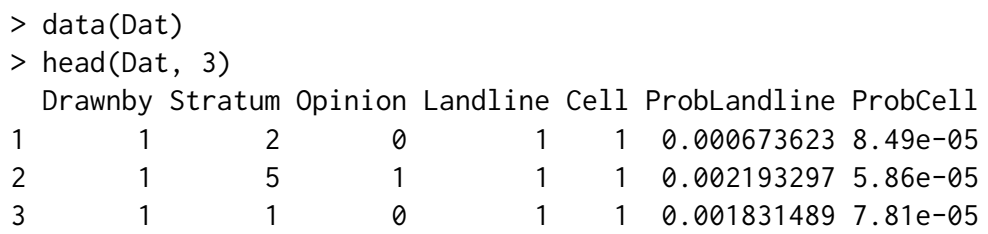

From the data of this survey we wish to estimate the number of people in Andalusia thinking that immigrants currently living in this region are quite a lot. In order to use functions of package Frames2, we need to split this dataset. The variables we will use to do this are Drawnby and Landline and Cell.

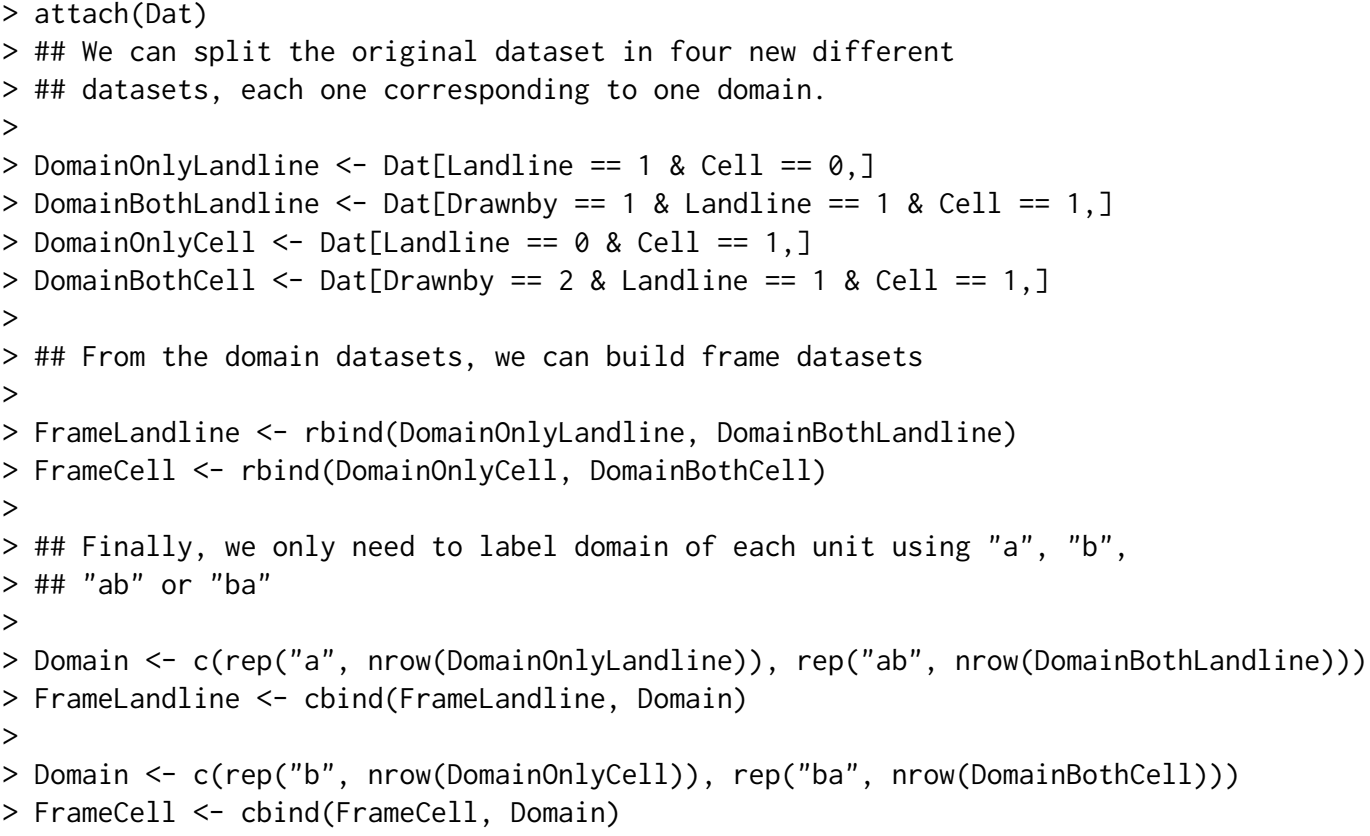

Now dual frame estimators, as PML estimator, can be computed:

$>$ summary (PML (FrameLandline\$Opinion, FrameCell\$Opinion, FrameLandline\$ProbLandline,

+ FrameCell\$ProbCell, FrameLandline\$Domain, FrameCell\$Domain, N_A $=4982920$,

$+\quad$ N_B $=$ 5707655))

Call:

$\operatorname{PML}(y s \mathrm{~A}=$ FrameLandline\$Opinion, $\mathrm{ysB}=$ FrameCell\$Opinion, pi_A = FrameLandline\$ProbLandline, pi_B = FrameCell\$ProbCell, domains_A = FrameLandline $\$$ Domain, domains_B = FrameCell\$Domain, N_A $=4982920$, N_B $=$ 5707655)

Estimation:

Total 3.231325e+06

Mean $4.635634 \mathrm{e}-01$

Variance Estimation: 
$[, 1]$

Var. Total 1.784362e+10

Var. Mean 3.672317e-04

Total Domain Estimations:

$[, 1]$

Total dom. a 219145.1

Total dom. ab 2318841.9

Total dom. b 1346646.1

Total dom. ba 1457501.0

Mean Domain Estimations:

$[, 1]$

Mean dom. a 0.4438149

Mean dom. ab 0.4990548

Mean dom. b 0.4172797

Mean dom. ba 0.4674919

Parameters:

gamma 0.3211534

As the overlap domain size is known, we can include additionally this information in the process and compute more accurate estimators as CalDF and CalSF.

$>$ summary (CalDF (FrameLandline\$Opinion, FrameCell\$Opinion, FrameLandline\$ProbLandline,

+ FrameCell\$ProbCell, FrameLandline\$Domain, FrameCell\$Domain, N_A $=4982920$,

$+\quad$ N_B $=5707655$, N_ab $=4339659)$ )

Call:

CalDF (ysA = FrameLandline\$0pinion, ysB = FrameCell\$0pinion,

pi_A = FrameLandline\$ProbLandline, pi_B = FrameCell $\$$ ProbCell,

domains_A $=$ FrameLandline\$Domain, domains_B $=$ FrameCell\$Domain,

N_A $=4982920$, N_B $=5707655, \quad$ N_ab $=4339659$ )

Estimation:

Total $2.985028 \mathrm{e}+06$

Mean $4.700153 \mathrm{e}-01$

Variance Estimation:

Var. Total $1.478990 \mathrm{e}+10$

Var. Mean 3.666844e-04

Parameters:

eta 0.7296841

$>$

$>$ summary(CalSF(FrameLandline\$Opinion, FrameCell\$Opinion, FrameLandline\$ProbLandline,

+ FrameCell\$ProbCell, FrameLandline\$ProbCell, FrameCell\$ProbLandline,

$+\quad$ FrameLandline\$Domain, FrameCell\$Domain, N_A $=4982920$, N_B $=5707655$,

$+\quad$ N_ab $=4339659)$ )

Call:

CalSF $(y s A=F r a m e L a n d l$ ine\$Opinion, $y s B=$ FrameCell\$Opinion,

pi_A = FrameLandline\$ProbLandline, pi_B = FrameCell\$ProbCell,

pik_ab_B = FrameLandline\$ProbCell, pik_ba_A = FrameCell\$ProbLandline,

domains_A $=$ FrameLandline\$Domain, domains_B $=$ FrameCell\$Domain, N_A $=4982920$,

N_B $=5707655, \quad$ N_ab $=4339659$ )

Estimation:

$[, 1]$

Total $2.986787 \mathrm{e}+06$

Mean $4.702923 \mathrm{e}-01$ 
Variance Estimation:

$[, 1]$

Var. Total 1.442969e+10

Var. Mean 3.577539e-04

Observe that the greater the information included in the estimation process is, the greater is the accuracy of the estimates.

\section{Summary}

The statistical literature about dual frame surveys started around 1960 and its development has evolved very quickly because these surveys are largely used by statistical agencies and private organizations to decrease sampling costs and to reduce frame undercoverage errors that could occur with the use of a single sampling frame.

Dual frame surveys can be more complicated to design and more complicated to analyze than those that use one frame only. There are several estimators of the population total available in the statistical literature. These estimators rely on weight adjustments to compensate for the multiplicity of the units in the overlap domain. Some of these estimators allow the handling of different types of auxiliary information at different levels. Nevertheless, none of the existing statistical software implements all of these estimators.

In this article we illustrate Frames2, a new $\mathrm{R}$ package for point and interval estimation in dual frame context. Functions in the package implement the most important estimators in the literature for population totals and means. We include two procedures (Pseudo-Empirical-Likelihood approach and calibration approach) to incorporate auxiliary information about frame sizes and also about one or several auxiliary variables in one or two frames. Post-stratification, raking ratio or regression estimation are all encompassed as particular cases of these estimation procedures. Additional functions for confidence interval estimation based on the jackknife variance estimation have been included as well.

The functionalities of the package Frames2 have been illustrated using several data sets DatA, DatB and Dat (included in the package) corresponding to different complex surveys. We envision future additions to the package that will allow for extensions to more than two frames.

Finally, we would like to direct the reader to the package vignettes named "est imation" (Estimation in a dual frame context) and "formatting. data" (Splitting and formatting data in a dual frame context) for further examples and background information.

\section{Acknowledgements}

This study was partially supported by Ministerio de Educación, Cultura y Deporte (grant MTM201235650 and FPU grant program, Spain), by Consejería de Economía, Innovación, Ciencia y Empleo (grant SEJ2954, Junta de Andalucía, Spain) and under the support of the project PRIN-SURWEY (grant 2012F42NS8, Italy). The authors are grateful to Manuel Trujillo, (IESA, Institute of Advanced Social Studies) for providing data and information about the OPIA (Opinions and Attitudes of the Andalusian Population regarding Immigration) survey. This paper and software have been considerably improved by the comments of the editor and the reviewers.

\section{Bibliography}

A. Alfons, J. Holzer, and M. Templ. laeken: Estimation of Indicators on Social Exclusion and Poverty, 2014. URL http: //CRAN. R-project.org/package=laeken. R package version 0.4.6. [p52]

A. Arcos, M. Rueda, M. G. Ranalli, and D. Molina. Frames2: Estimation in Dual Frame Surveys, 2015. URL http://CRAN.R-project.org/package=Frames2. R package version 0.1.1. [p53]

M. D. Bankier. Estimators based on several stratified samples with applications to multiple frame surveys. Journal of the American Statistical Association, 81(396):1074-1079, 1986. [p54, 55]

J. C. Deville. Estimation de la variance pour les enquêtes en deux phases. Manuscript, INSEE, Paris, 1993. $[\mathrm{p} 56,65]$

W. A. Fuller and L. F. Burmeister. Estimation for samples selected from two overlapping frames. In ASA Proceedings of the Social Statistics Sections, pages 245-249, 1972. [p54, 55] 
W. A. Fuller, W. Kennedy, D. Schell, G. Sullivan, and H. J. Park. PCCARP. Iowa State University Statistical Laboratory, 1989. [p52]

H. A. Gutierrez Rojas. TeachingSampling: Selection of Samples and Parameter Estimation in Finite Population, 2014. URL http://CRAN.R-project.org/package=TeachingSampling. R package version 3.2.1. [p52]

H. O. Hartley. Multiple frame surveys. In Proceedings of the American Statistical Association, Social Statistics Sections, pages 203-206, 1962. [p53, 54]

H. O. Hartley. Multiple frame methodology and selected applications. Sankhyā C, 36(3):99-118, 1974. [p54]

IBM Corporation. IBM SPSS Statistics for Windows, Version 22.0. Armonk, NY, 2013. URL http: //www.ibm.com/software/analytics/spss/. [p52]

G. Kalton and D. W. Anderson. Sampling rare populations. Journal of the Royal Statistical Society A, 149 (1):65-82, 1986. [p54]

S. Lohr and J. Rao. Inference in dual frame surveys. Journal of the American Statistical Association, 95 (449):271-280, 2000. [p56]

T. Lumley. survey: Analysis of Comples Survey Samples, 2014. URL http://CRAN.R-project.org/ package=survey. R package version 3.30. [p52]

M. H. Quenouille. Problems in plane sampling. The Annals of Mathematical Statistics, 20(3):355-375, 1949. [p56]

M. H. Quenouille. Notes on bias in estimation. Biometrika, 43(3/4):353-360, 1956. [p56]

M. G. Ranalli, A. Arcos, M. Rueda, and A. Teodoro. Calibration estimators in dual frames surveys. arXiv:1312.0761 [stat.ME], 2013. [p56]

J. N. K. Rao and C. J. Skinner. Estimation in dual frame surveys with complex designs. In Proceedings of the Survey Method Section, Statistical Society of Canada, pages 63-68, 1996. [p55]

J. N. K. Rao and C. Wu. Pseudo empirical likelihood inference for multiple frame surveys. Journal of the American Statistical Association, 105(492):1494-1503, 2010. [p55, 62]

Research Triangle Institute. SUDAAN, Version 11.0.1. Research Triangle Park, NC, 2013. URL http://www.rti.org/sudaan/. [p52]

SAS Institute Inc. SAS Software, Version 9.4. Cary, NC, 2013. URL http://www. sas. com/. [p52]

C. J. Skinner. On the efficiency of raking ratio estimation for multiple frame surveys. Journal of the American Statistical Association, 86(415):779-784, 1991. [p55]

C. J. Skinner and J. N. K. Rao. Estimation in dual frame surveys with complex designs. Journal of the American Statistical Association, 91(443):349-356, 1996. [p55]

Stata Corporation. Stata Statistical Software, Version 14.0. College Station, TX, 2015. URL http: //www. stata.com/. [p52]

Systat Software Inc. Systat, Version 13.0. San Jose, California, 2009. URL http://www. systat. com. [p52]

M. Templ. CRAN Task View: Official Statistics and Survey Methodology, 2014. URL http://CRAN. Rproject . org/view=0fficialStatistics. [p52]

Y. Tillé and A. Matei. sampling: Survey Sampling, 2012. URL http://CRAN. R-project.org/package= sampling. R package version 2.5. [p52, 64]

K. M. Wolter. Introduction to Variance Estimation. Springer, New York, 2nd edition, 2007. [p56]

C. Wu. Algorithms and R codes for the pseudo empirical likelihood method in survey sampling. Survey Methodology, 31(2):239-243, 2005. [p55, 64] 
Antonio Arcos

Department of Statistics and Operational Research University of Granada

Spain

arcos@ugr.es

David Molina

Department of Statistics and Operational Research University of Granada

Spain

dmolinam@ugr.es

Maria Giovanna Rannalli

Department of Political Science

University of Perugia

Italy

giovanna.ranalli@stat.unipg. it

María del Mar Rueda

Department of Statistics and Operational Research University of Granada

Spain

mrueda@ugr.es 